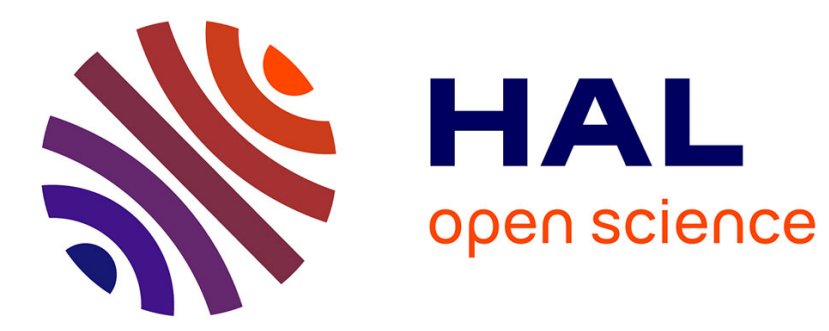

\title{
Global and Koopman modes analysis of sound generation in mixing layers
}

Ge Song, Frédéric Alizard, Jean-Christophe Robinet, Xavier Gloerfelt

\section{To cite this version:}

Ge Song, Frédéric Alizard, Jean-Christophe Robinet, Xavier Gloerfelt. Global and Koopman modes analysis of sound generation in mixing layers. Physics of Fluids, 2013, 25, pp.124101. 10.1063/1.4834438 . hal-01069675

\section{HAL Id: hal-01069675 \\ https://hal.science/hal-01069675}

Submitted on 29 Sep 2014

HAL is a multi-disciplinary open access archive for the deposit and dissemination of scientific research documents, whether they are published or not. The documents may come from teaching and research institutions in France or abroad, or from public or private research centers.
L'archive ouverte pluridisciplinaire HAL, est destinée au dépôt et à la diffusion de documents scientifiques de niveau recherche, publiés ou non, émanant des établissements d'enseignement et de recherche français ou étrangers, des laboratoires publics ou privés. 


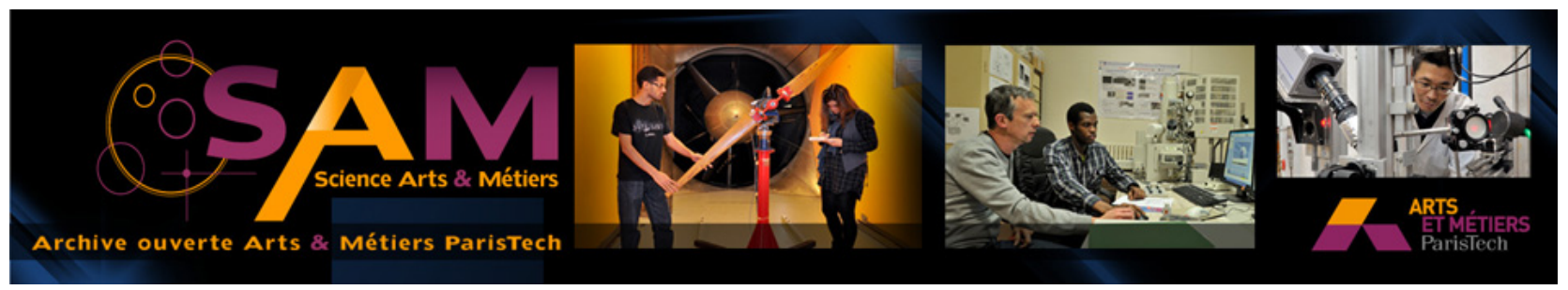

Science Arts \& Métiers (SAM)

is an open access repository that collects the work of Arts et Métiers ParisTech researchers and makes it freely available over the web where possible.

This is an author-deposited version published in: http://sam.ensam.eu

Handle ID: .http://hdl.handle.net/10985/8642

\section{To cite this version :}

Ge SONG, Frédéric ALIZARD, Jean-Christophe ROBINET, Xavier GLOERFELT - Global and Koopman modes analysis of sound generation in mixing layers - Physics of Fluids - Vol. 25, p.124101- 2013 


\section{Global and Koopman modes analysis of sound generation in mixing layers}

G. Song $^{+}$, F. Alizard ${ }^{\dagger}$, J.-C. Robinet ${ }^{+}$, X. Gloerfelt ${ }^{+1}$

Laboratoire DynFluid, Arts et Métiers ParisTech ${ }^{+}$and $C N A M^{\dagger}$, 151 Boulevard de l'Hopital 75013 Paris.

(Dated: 29 September 2014)

It is now well established that linear and nonlinear instability waves play a significant role in the noise generation process for a wide variety of shear flows such as jets or mixing layers. In that context, the problem of acoustic radiation generated by spatially growing instability waves of two-dimensional subsonic and supersonic mixing layers are revisited in a global point of view, i.e. without any assumption about the base flow, in both a linear and a nonlinear framework by using global and Koopman mode decompositions. In that respect, a timestepping technique based on disturbance equations is employed to extract the most dynamically relevant coherent structures for both linear and nonlinear regimes. The present analysis proposes thus a general strategy for analysing the near-field coherent structures which are responsible for the acoustic noise in these configurations. In particular, we illustrate the failure of linear global modes to describe the noise generation mechanism associated with the vortex pairing for the subsonic regime whereas they appropriately explain the Mach wave radiation of instability waves in the supersonic regime. By contrast, the Dynamic Mode Decomposition (DMD) analysis captures both the near-field dynamics and the far-field acoustics with a few number of modes for both configurations. In addition, the combination of DMD and linear global modes analyses provides new insight about the influence on the radiated noise of nonlinear interactions and saturation of instability waves as well as their interaction with the mean flow.

PACS numbers: 47.20.Ky, 43.28.+h, 45.30.+s, 47.20.Ft

Keywords: global linear stability, computational aeroacoustics, modal decomposition, Koopman modes, mixing layers 
Global and Koopman modes analysis of sound generation in mixing layers

\section{INTRODUCTION}

Sound generation in a flow is of key importance for a wide range of industrial applications, such as noise in aircraft or ground transport. In such configurations, inhomogeneities in the aerodynamic field are most often at the origin of the sound. This is known as aeroacoustics. Strong gradient zones where the flow is sheared and distorted are generally associated with a complex dynamics, leading eventually to a large range of time and spatial scales in which successive bifurcations may lead to turbulence. This vortex dynamics is also usually responsible for significant acoustical radiation in the far field. Nevertheless, from the point of view of instability theory, vortical structures may be regarded in Fourier-Laplace space as a collection of instability waves with distinct frequencies and wavelengths. In the compressible framework, the vortex dynamics can generate sound. How then connect the instability waves to acoustic radiation? As a canonical flow model to investigate flow noise mechanisms, the shear layer has been successfully used in computational aeroacoustics. The shear layer is thus a good prototype for studying the role of instabilities in the mechanism of acoustic emission. The objective of this paper is to propose a modern computation method of instabilities that could potentially be applied to complex flows and also to show that this method calculates the resulting acoustic radiation. Two shear layers, one subsonic and the other supersonic, as in the study by Cheung \& Lele ${ }^{8}$, are reviewed in the context of global instability.

The laminar free shear layer has been extensively investigated both from stability $4,5,24,36$ or numerical simulation ${ }^{27,34,39}$ points of view for various flow regimes (incompressible, subsonic or supersonic). The study of the associated acoustic radiation by numerical simulations was undertaken in the $90 \mathrm{~s}$ by Colonius et al. ${ }^{10}$ and Bogey \& Bailly ${ }^{7}$ for the two-dimensional mixing layer. However, the link between the development of instability waves and acoustic radiation is not yet directly studied.

The first analyzes linking instabilities to the acoustic radiation for a shear layer were made by Tam \& Morris ${ }^{49}$ and Tam \& Burton ${ }^{47}$ using inviscid local and parallel stability theory with an asymptotic expansion. The evolution of acoustic waves in the far field was adequately described. All these studies on this subject have shown that linear stability analysis did not allow to capture the acoustic radiation for a free shear layer in the subsonic regime. It is thus necessary to take into account nonlinear processes. For a supersonic shear 
Global and Koopman modes analysis of sound generation in mixing layers

layer, on the contrary, the linear stability theory predicts well the Mach wave radiation. Cheung \& Lele ${ }^{8}$ extended these approaches by studying the non-parallel effects as well as the nonlinear regime with the linear and nonlinear Parabolized Stability Equations (PSE) on the near-field dynamics and the resulting acoustic radiation of two-dimensional compressible shear layers.

They also showed that nonlinear PSE method could predict the nonlinear dynamics in the near field, and in particular the vortex pairings observed in a subsonic shear layer. Although the vortex-pairing sound can be accurately captured in the near field, the linear or nonlinear PSE methods do not properly restitute the acoustic field in the far field. This limitation is mainly due to the homogeneous boundary conditions imposed along the normal direction. Another disadvantage of PSE methods, assuming that the base flow is weakly non-parallel, is its inability to capture upstream-propagating waves that may be present in many practical applications such as cavity flows ${ }^{19}$ or the impinging jet ${ }^{23}$.

To address these limitations, it is necessary to analyze the dynamics through global mode decomposition. This approach has now become classic in the incompressible regime ${ }^{12,51,52}$ but still uncommon for compressible flows and more specifically for the supersonic regime or when the associated acoustic radiation is considered. From a methodological point of view, there are two families of methods for computing global modes. The first approach is a matrix-forming method, which consists in computing the eigenvalues of the Jacobian matrix from the linearized Navier-Stokes equations. This method is usually carried out in four steps: (i) establishment of linearized Navier-Stokes (LDE), (ii) Laplace transform of LDE, (iii) spatial discretization of resulting stability equation, (iv) resolution of the resulting eigenproblem. A number of problems is raised in the compressible regime especially when the phase velocity of the mode is locally supersonic with respect to the base flow. Although some problems can be partially solved by reversing steps (ii) and (iii), this method requires the storage of a large matrix, which limits to relatively simple and two-dimensional (for the base flow) configurations. Recent applications of this method in the compressible regime can be found in Kierkegaard et al. ${ }^{25}$ and Yamouni et al. ${ }^{53}$ for open cavities, Fosas de Pando et al. ${ }^{13}$ for airfoil flow, Nichols \& Lele ${ }^{35}$ and Garnaud et al. ${ }^{17,18}$ for jet flows and Mack \& Schmid $^{30}$ for swept parabolic flow. In short, a critical step in the process of systematically investigating stability properties by matrix-forming methods is to solve very large eigenvalue problems and to be uniformly valid with respect to the boundary conditions. 
Global and Koopman modes analysis of sound generation in mixing layers

The second approach is a matrix-free method, in which very large eigenvalue problems are solved by storing only velocity fields at different times instead of large matrices. This socalled time-stepper technique was popularized in fluid mechanics by Edwards et al. ${ }^{15}$ and recently used for Blasius flow by Bagheri et al. ${ }^{3}$. There are two main advantages associated with this method. On one hand, its cost. The modes are calculated using a matrix whose size is the number of snapshots. Also, management of boundary conditions for the disturbances is facilitated; it is thus possible to apply nonreflecting boundary conditions compatible with the acoustic wave radiation. On the other hand, its extension to the nonlinear regime is straightforward. To achieve this, it is sufficient to compute Koopman modes ${ }^{31,38,41}$ instead of global modes by replacing the linearized Navier-Stokes solver by the full nonlinear solver.

The objective of the paper is to characterize the relationship between development of instabilities and the resulting acoustic radiation within a global instability framework. Wave effects of linear and nonlinear instability on the near- and far-field dynamics of twodimensional compressible free shear layers are studied. Two shear layers are considered, similar to those analyzed by Cheung \& Lele ${ }^{8}$. A subsonic configuration, forced by the fundamental frequency and its first sub-harmonic, where nonlinear mechanisms are responsible for the acoustic radiation (vortex pairing sound) and a supersonic configuration, forced only by its fondamental, where the sound is produced by Mach wave radiation and mainly caused by linear instabilities.

From a methodological point of view, a nonlinear disturbance equations (NLDE) formulation of two-dimensional compressible Navier-Stokes equations is used. This approach is commonly used for aeroacoustic computations, see Morris et al. ${ }^{32,33}$. The advantage of this formulation is firstly that the base flow is imposed and secondly it is very easy to move from linear to nonlinear equations. Based on the linear or nonlinear version of the code, global or Koopman modes are then extracted by a matrix-free method.

The paper is organized as follows. In section II, the direct, NLDE numerical methods and algorithms to extract the global and Koopman modes are presented. Section III is dedicated to the subsonic shear layer where the aerodynamic and the acoustic fields are computed in $\S \mathrm{A}$. In particular, the NLDE solver is validated against the direct solver and the base flow is computed. The global modes $(\S \mathrm{B})$ and the Koopman modes $(\S \mathrm{C})$ are extracted by the time-stepping method and then compared to the NLDE solutions. Special attention is paid to the reconstruction of the solution and its associated acoustic radiation. The supersonic 
Global and Koopman modes analysis of sound generation in mixing layers

shear layer case is discussed in section IV in the same way as the subsonic case. Finally, Section V provides concluding remarks.

\section{NUMERICAL METHODS AND ALGORITHMS}

\section{A. Direct solvers}

\section{Full Navier-Stokes equations}

The two-dimensional compressible Navier-Stokes equations along with the ideal gas equation of state are used as a mathematical model to describe the dynamics of the mixing layer. The governing equations in conservative form may be written in a Cartesian frame as

$$
\frac{\partial \mathbf{U}}{\partial t}+\frac{\partial \mathbf{E}_{\mathbf{e}}}{\partial x}+\frac{\partial \mathbf{F}_{\mathbf{e}}}{\partial y}-\frac{\partial \mathbf{E}_{\mathbf{v}}}{\partial x}-\frac{\partial \mathbf{F}_{\mathbf{v}}}{\partial y}=0
$$

$\mathbf{U}$ is the vector of conservative variables $\mathbf{U}=(\rho, \rho u, \rho v, e)^{T}$, where $\rho$ is the fluid density and $u, v$ are the velocity components in the two directions. The total energy per unit volume of fluid is defined as

$$
e=\rho\left[c_{v} T+\frac{1}{2}\left(u^{2}+v^{2}\right)\right]=\frac{p}{\gamma-1}+\frac{1}{2} \rho\left(u^{2}+v^{2}\right)
$$

where $p, T, c_{v}$ and $\gamma$ are the pressure, temperature, specific heat at constant volume and specific ratio, respectively. The ratio of specific heats $\gamma=c_{p} / c_{v}$ is taken to be 1.4. Note that, in (2), the ideal gas law $p=\rho r T$ is used to relate between the thermodynamic variables, where the gas constant $r$ is fixed to $287.06 \mathrm{~J} \cdot \mathrm{kg}^{-1} \cdot \mathrm{K}^{-1}$. The specific heat at constant pressure $c_{p}$ and specific heat at constant volume $c_{v}$ are defined as $c_{v}=c_{p} / \gamma$ and $c_{p}=r \gamma /(\gamma-1)$. $\mathbf{E}_{\mathbf{e}}$ and $\mathbf{F}_{\mathbf{e}}$ are the inviscid convective fluxes in the two directions, while $\mathbf{E}_{\mathbf{v}}$ and $\mathbf{F}_{\mathbf{v}}$ are the fluxes including both viscous stresses and thermal conduction:

$$
\begin{aligned}
& \mathbf{E}_{\mathbf{e}}=\left(\rho u, \rho u^{2}+p, \rho u v,(e+p) u\right)^{T} \\
& \mathbf{F}_{\mathbf{e}}=\left(\rho v, \rho u v, \rho v^{2}+p,(e+p) v\right)^{T} \\
& \mathbf{E}_{\mathbf{v}}=\left(0, \tau_{x x}, \tau_{x y}, u \tau_{x x}+v \tau_{x y}-q_{x}\right)^{T} \\
& \mathbf{F}_{\mathbf{v}}=\left(0, \tau_{y x}, \tau_{y y}, u \tau_{y x}+v \tau_{y y}-q_{y}\right)^{T}
\end{aligned}
$$

The viscous stress tensor is defined for a Newtonian fluid with Stokes' hypothesis as

$$
\tau_{x x}=\mu\left(\frac{4}{3} \frac{\partial u}{\partial x}-\frac{2}{3} \frac{\partial v}{\partial y}\right) ; \tau_{x y}=\tau_{y x}=\mu\left(\frac{\partial u}{\partial y}+\frac{\partial v}{\partial x}\right) ; \tau_{y y}=\mu\left(\frac{4}{3} \frac{\partial v}{\partial y}-\frac{2}{3} \frac{\partial u}{\partial x}\right)
$$


Global and Koopman modes analysis of sound generation in mixing layers

The dynamic molecular viscosity $\mu$ is approximated with Sutherland's law:

$$
\mu(T)=\mu_{0}\left(\frac{T}{T_{0}}\right)^{\frac{3}{2}} \frac{T_{0}+110.4}{T+110.4}
$$

with the reference point $T_{0}=273.15 \mathrm{~K}$ and $\mu_{0}=1.711 \times 10^{5} \mathrm{~kg} \cdot \mathrm{m}^{-1} \cdot \mathrm{s}^{-1}$. The heat flux components model thermal conduction with Fourier's law:

$$
q_{x}=-\kappa \frac{\partial T}{\partial x} ; q_{y}=-\kappa \frac{\partial T}{\partial y}
$$

where $\kappa$ is the coefficient of thermal conductivity. The Prandtl number is $\operatorname{Pr}=\mu c_{p} / \kappa$ and we assume a constant value of $\operatorname{Pr}=0.723$.

\section{Nonlinear disturbance equations}

Nonlinear disturbance equations (NLDE) have first been proposed by Morris et al. ${ }^{32}$. By decomposing the instantaneous flow variable into a mean flow and fluctuating part, they obtained the disturbance equations. In their work, the mean flow are calculated with a Reynolds averaged Navier-Stokes (RANS) solver, and the perturbations quantities are determined directly. This NLDE approach has been used rather satisfactorily to calculate the acoustic fields in a supersonic jet ${ }^{33}$ or a hot jet ${ }^{28}$.

In the present stability study, a perturbative version of the Navier-Stokes equations allows a better control of the base flow and the investigation of the linear regime by simply omitting the nonlinear terms in the equations. To investigate the behavior of perturbations about the base flow, the flow vector $\mathbf{q}$ is decomposed into the steady base flow $\mathbf{q}_{b}$ and a perturbation $\mathbf{q}^{\prime}$ :

$$
\mathbf{q}=\mathbf{q}_{b}+\mathbf{q}^{\prime}
$$

Note that the base flow is non-bifurcated and an equilibrium solution of $(1)^{45}$. Substitution of (3) into (1) results in a set of base flow and perturbation terms. After rearrangement of these terms, the NLDE in conservative form can be written as

$$
\frac{\partial \mathbf{U}^{\prime}}{\partial t}+\frac{\partial \mathbf{E}_{\mathbf{e}}{ }^{\prime}}{\partial x}+\frac{\partial \mathbf{F}_{\mathbf{e}}{ }^{\prime}}{\partial y}-\frac{\partial \mathbf{E}_{\mathbf{v}}{ }^{\prime}}{\partial x}-\frac{\partial \mathbf{F}_{\mathbf{v}}{ }^{\prime}}{\partial y}=0
$$

where $\mathbf{U}^{\prime}=\left(\rho^{\prime}, \rho_{b} u^{\prime}+\rho^{\prime} u_{b}+\rho^{\prime} u^{\prime}, \rho_{b} v^{\prime}+\rho^{\prime} v_{b}+\rho^{\prime} v^{\prime}, e^{\prime}\right)^{T}$. The convective perturbation fluxes 
Global and Koopman modes analysis of sound generation in mixing layers

$\mathbf{E}_{\mathbf{e}}{ }^{\prime}$ and $\mathbf{F}_{\mathbf{e}}{ }^{\prime}$ containing both linear and nonlinear terms are given by

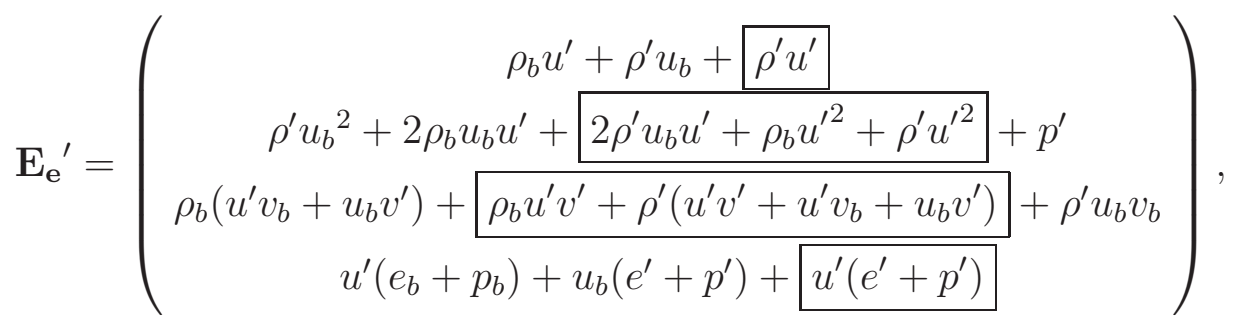

$$
\begin{aligned}
& \mathbf{F}_{\mathbf{e}}{ }^{\prime}=\left(\begin{array}{c}
\rho v^{\prime}+\rho^{\prime} v_{b}+\rho^{\prime} v^{\prime} \\
\rho_{b}\left(u_{b} v^{\prime}+v_{b} u^{\prime}\right)+\rho_{b} u^{\prime} v^{\prime}+\rho^{\prime}\left(u_{b} v^{\prime}+v_{b} u^{\prime}+u^{\prime} v^{\prime}\right) \\
\rho^{\prime} v_{b}{ }^{2}+2 \rho_{b} v_{b} v^{\prime}+2 \rho^{\prime} v_{b} v^{\prime}+\rho_{b} v^{\prime 2}+\rho^{\prime} v^{\prime 2} \\
v^{\prime}\left(e_{b}+p_{b}\right)+v_{b}\left(e^{\prime}+p^{\prime}\right)+p^{\prime}\left(e^{\prime}+p^{\prime}\right)
\end{array}\right),
\end{aligned}
$$

with $\bullet$ : the nonlinear terms

and the viscous perturbation stresses $\mathbf{E}_{\mathbf{v}}{ }^{\prime}$ and $\mathbf{F}_{\mathbf{v}}{ }^{\prime}$ are given by

$$
\begin{aligned}
& \mathbf{E}_{\mathbf{v}}{ }^{\prime}=\left(0, \tau_{x x}^{\prime}, \tau_{x y}^{\prime}, u^{\prime} \tau_{x x}^{b}+u^{\prime} \tau_{x x}^{\prime}+v^{\prime} \tau_{x y}^{b}+v^{\prime} \tau_{x y}^{\prime}+u_{b} \tau_{x x}^{\prime}+v_{b} \tau_{x y}^{\prime}-q_{x}{ }^{\prime}\right) \\
& \mathbf{F}_{\mathbf{v}}{ }^{\prime}=\left(0, \tau_{y x}^{\prime}, \tau_{y y}^{\prime}, u^{\prime} \tau_{y x}^{b}+u^{\prime} \tau_{y x}^{\prime}+v^{\prime} \tau_{y y}^{b}+v^{\prime} \tau_{y y}^{\prime}+u_{b} \tau_{y x}^{\prime}+v_{b} \tau_{y y}^{\prime}-q_{y}{ }^{\prime}\right)
\end{aligned}
$$

The components of the perturbation viscous stress tensor are:

$$
\tau_{x x}^{\prime}=\mu\left(\frac{4}{3} \frac{\partial u^{\prime}}{\partial x}-\frac{2}{3} \frac{\partial v^{\prime}}{\partial y}\right) ; \tau_{x y}^{\prime}=\tau_{y x}^{\prime}=\mu\left(\frac{\partial u^{\prime}}{\partial y}+\frac{\partial v^{\prime}}{\partial x}\right) ; \tau_{y y}^{\prime}=\mu\left(\frac{4}{3} \frac{\partial v^{\prime}}{\partial y}-\frac{2}{3} \frac{\partial u^{\prime}}{\partial x}\right)
$$

Similarly for the components of the base-flow viscous stress tensor ${ }^{9}$ :

$$
\tau_{x x}^{b}=\mu\left(\frac{4}{3} \frac{\partial u_{b}}{\partial x}-\frac{2}{3} \frac{\partial v_{b}}{\partial y}\right) ; \tau_{x y}^{b}=\tau_{y x}^{b}=\mu\left(\frac{\partial u_{b}}{\partial y}+\frac{\partial v_{b}}{\partial x}\right) ; \tau_{y y}^{b}=\mu\left(\frac{4}{3} \frac{\partial v_{b}}{\partial y}-\frac{2}{3} \frac{\partial u_{b}}{\partial x}\right)
$$

and the perturbation heat flux components are written as

$$
q_{x}^{\prime}=-\kappa \frac{\partial T^{\prime}}{\partial x} ; q_{y}^{\prime}=-\kappa \frac{\partial T^{\prime}}{\partial y}
$$

As soon as the conservative perturbation vector $\mathbf{U}^{\prime}$ is obtained from the solution of (4), the velocity perturbation $u^{\prime}, v^{\prime}$ may be obtained from the definition of $\mathbf{U}^{\prime}$ and the base flow values, while the fluctuating pressure $p^{\prime}$ may be deduced from

$$
\begin{aligned}
& e^{\prime}=\frac{p^{\prime}}{\gamma-1}+\rho_{b}\left(u^{\prime} u_{b}+v^{\prime} v_{b}\right)+\rho^{\prime}\left(u^{\prime} u_{b}+v^{\prime} v_{b}\right) \\
& +\frac{1}{2} \rho_{b}\left(u^{\prime 2}+v^{\prime 2}\right)+\frac{1}{2} \rho^{\prime}\left(u_{b}^{2}+v_{b}^{2}\right)+\frac{1}{2} \rho^{\prime}\left(u^{\prime 2}+v^{\prime 2}\right)
\end{aligned}
$$


Global and Koopman modes analysis of sound generation in mixing layers

\section{Algorithm and boundary treatment}

Two main difficulties have to be considered when writing a computational aeroacoustics (CAA) code: to propose an efficient algorithm for the acoustic wave propagation and to use accurate boundary conditions for the truncated computional domain. In fact, acoustic waves have some typical features that should be taken into account: their nondissipative and nondispersive character, the extremely low amplitude of acoustic perturbations, and the need to resolve high frequencies accurately. Therefore, the NLDE are advanced in time using an explicit six-substep Runge-Kutta scheme, with coefficients optimized in the frequency space and fourth-order formal accuracy ${ }^{6}$. An 11-point-stencil centered DispersionRelation Preserving (DRP) scheme, initially developed by Tam \& Webb ${ }^{50}$, is used to obtain the spatial derivatives. To avoid the so-called grid-to-grid oscillations due to the centered finite-difference scheme, an 11-point-stencil centered selective filter is introduced in order to filter out non-physical high-frequency oscillations ${ }^{6}$.

Non-reflecting conditions are required to mimic an infinite continuous medium. To this end, a set of radiation and outflow boundary conditions, proposed by Tam \& Dong ${ }^{48}$, are used. In particular, a sponge zone combining grid stretching and a Laplacian filter is applied at the outflow boundary ( $\mathrm{see}^{21}$ for details).

To trigger the convective flow instabilities, a forcing is introduced in the inlet plane. In particular, for the inlet condition, the radiation conditions, expressed in the polar coordinates $(r, \theta)$ centered at the center of the computational domain, are modified at the inlet boundary as

$$
\left(\frac{1}{V_{g}} \frac{\partial}{\partial t}+\frac{\partial}{\partial r}+\frac{1}{r}\right)\left[\begin{array}{c}
\rho-\rho_{b} \\
u-u_{b} \\
v-v_{b} \\
p-p_{b}
\end{array}\right]=\left(\frac{1}{V_{g}} \frac{\partial}{\partial t}+\frac{\partial}{\partial r}+\frac{1}{r}\right)\left[\begin{array}{c}
\rho_{i n}^{\prime} \\
u_{i n}^{\prime} \\
v_{i n}^{\prime} \\
p_{i n}^{\prime}
\end{array}\right]
$$

where $V_{g}=u_{b} \cos \theta+v_{b} \sin \theta+\sqrt{c_{b}^{2}-\left(v_{b} \cos \theta-u_{b} \sin \theta\right)^{2}}$ denotes the group velocity of acoustic waves, $c_{b}$ being the local sound speed calculated from base-flow values. The vector $\mathbf{q}_{i n}^{\prime}=\left(\rho_{i n}^{\prime}, u_{i n}^{\prime}, v_{i n}^{\prime}, p_{i n}^{\prime}\right)^{T}$ assembles the density, velocity components, and pressure fluctuations to be imposed at the inflow. It is explicitely defined by the solutions of local linear stability analysis as described in Appendix A, so the right-hand side of (6) is known. 
Global and Koopman modes analysis of sound generation in mixing layers

\section{B. Global and Koopman modes}

\section{Iteratives techniques based on snapshots}

In classical dynamical system theory, the evolution of flow variables $\mathbf{q}^{\prime}$ defined on a state space $\mathcal{D}$ may be expressed as

$$
\frac{\partial \mathbf{q}^{\prime}}{\partial t}=\mathcal{F}\left(\mathbf{q}^{\prime}\right)
$$

From a time-stepper point of view, the system (7) is rewritten as

$$
\mathbf{q}^{\prime}(t+\Delta t)=\mathcal{B}(\Delta t) \mathbf{q}^{\prime}(t)
$$

where $t$ represents the time evolution and $\mathcal{B}(\Delta t)$ is a propagator for a time step $\Delta t$. In this context, a linear snapshot-to-snapshot mapping is associated with $\mathcal{B}(\Delta t)$, where a data sequence is generated by the time integration of the dynamical system (7). The Nyquist criterion applied to $\Delta t$ determines which structures are solved in the frequency domain.

In a time-stepper framework, the coherent structures are associated with eigenmodes of $\mathcal{B}(\Delta t)$, called the Ritz eigenvalues. Hence, this theory can be applied in both a linear and nonlinear framework.

Let us consider a set of $N$ snapshots of (7), separated in time by $\Delta t$

$$
\mathcal{S}^{N}=\left(S_{1}, S_{2}, \ldots S_{N}\right)
$$

where the propagator $\mathcal{B}(\Delta t)$ maps an instantaneous $S_{i}$ onto the next one $S_{i+1}$. In a linear theory, $\mathcal{B}(\Delta t)$ is linked to the Jacobian matrix about a steady state, referred to as $\mathcal{A}$, via

$$
\mathcal{B}(\Delta t)=e^{\mathcal{A}(\Delta t)}
$$

The eigenmodes of the propagator yield the so-called global modes. In a nonlinear framework, the eigenmodes of $\mathcal{B}(\Delta t)$ are connected to the Koopman modes (see Schmid et al. ${ }^{43}$ and Mezic $^{31}$ ). Arnoldi-type algorithms are therefore suitable for obtaining an approximation of the dominant eigenmodes of the propagator by using (9).

In a linear framework, an orthonormalized basis $\left(\mathcal{S}^{N}\right)^{\perp}$ of $\mathcal{S}^{N}$ is constructed using a modified Gram-Schmidt orthogonalization algorithm. A projection of $\mathcal{B}(\Delta t)$ onto $\left(\mathcal{S}^{N}\right)^{\perp}$ yields the system:

$$
\mathcal{B}(\Delta t)\left(\mathcal{S}^{N}\right)^{\perp}=\left(\mathcal{S}^{N}\right)^{\perp} \mathbf{H}
$$


Global and Koopman modes analysis of sound generation in mixing layers

where $\mathbf{H}$ is an $N \times N$ upper Hessenberg matrix. By increasing the number of snapshots $N$, the eigenmodes of $\mathbf{H}$ converge toward the dominant eigenmodes of $\mathcal{B}(\Delta t)$.

In a nonlinear framework, the Dynamic Mode Decomposition algorithm yields an approximation of the Koopman modes ${ }^{41}$. The $N$-th snapshot is expressed as a linear combination of $\left(S_{1}, S_{2}, \ldots, S_{N-1}\right)$. The projection of $\mathcal{B}(\Delta t)$ onto $\mathcal{S}^{N}$ leads thus to

$$
\mathcal{B}(\Delta t)\left(\mathcal{S}^{N}\right)=\left(\mathcal{S}^{N}\right) \mathbf{C}
$$

with $\mathbf{C}$ an $N \times N$ companion matrix, whose eigenvalues approximate the dominant Koopman modes. In this context, the expression 'dominant modes' is used for the most observable modes. One may remark that the algorithm associated with the global modes could be recovered from a QR decomposition of the companion matrix $\mathbf{C}$.

This Arnoldi-type method allows us to extract the most dynamically relevant coherent structures of linear and nonlinear dynamics. These modes have the property that they attribute to each coherent structure a spatial shape, $\hat{\mathbf{q}}_{\mathbf{k}}$, multiplied by a time-dependent function of the form $e^{-i \omega^{k} t}$ with $\omega^{k}=\omega_{r}^{k}+i \omega_{i}^{k}\left(\omega_{r}^{k}\right.$ its circular frequency and $\omega_{i}^{k}$ its temporal amplification rate) and such that

$$
\lambda^{k}=\frac{\log \left(\omega^{k}\right)}{\Delta t}
$$

where $\lambda^{k}$ are the eigenvalues of $\mathcal{B}(\Delta t)$.

As a consequence, a similar numerical method, based on data-sequence of snapshots is employed in the following to treat both linear and nonlinear dynamics. More details about the linear algorithm can be found $i^{2}$.

One may remark that each method requires an orthogonal projection onto a data sequence. Our particular interest is to deal with compressible flow and aeroacoustics. Then, we introduce a compressible inner product $(\bullet, \bullet)_{E}$ that includes both kinetic and internal energies (11)

$$
\left(\mathbf{q}_{1}^{\prime}, \mathbf{q}_{2}^{\prime}\right)_{E}=\int_{\Omega}\left[\frac{1}{2} \rho_{b}\left(u_{1}^{\prime *} u_{2}^{\prime}+v_{1}^{\prime *} v_{2}^{\prime}\right)+\frac{p_{1}^{\prime *} p_{2}^{\prime}}{p_{b}(\gamma-1)}\right] d V
$$

where $\Omega$ represents the computational domain, $\bullet_{b}$ refers to the basic state, and $*$ denotes the complex conjugate. 
Global and Koopman modes analysis of sound generation in mixing layers

\section{Orthogonal projection}

Both global and Koopman modes are not necessarily orthogonal. In order to perform a projection of a solution of our dynamical system (7) onto a set of eigenmodes of the propagator, a modified Gram-Schmidt procedure is undertaken to orthogonalize the basis $\left(\operatorname{see}^{1}\right.$ and $\left.^{16}\right)$. Let us denote the orthogonal basis as $\left(\mathbf{q}_{0}^{\prime \perp}, \mathbf{q}_{1}^{\prime \perp}, \cdots, \mathbf{q}_{m-1}^{\prime \perp}\right)$. An instantaneous field may be expanded as

$$
\mathbf{q}^{\prime}(\mathbf{x})=\sum_{k=0}^{m-1} \xi_{k} \mathbf{q}_{k}^{\prime \perp}(\mathbf{x})
$$

Taking the orthogonality of the basis into consideration,

$$
\xi_{k}=\left(\mathbf{q}_{k}^{\prime \perp}, \mathbf{q}_{0}^{\prime}\right)_{E}
$$

Therefore, the coordinates in the basis of global/Koopman modes, referred to as $K_{k}$, are recovered by making use of a matrix product:

$$
\mathbf{K}=\mathcal{P}^{-1} \xi
$$

with $\mathbf{K}=\left(K_{0}, K_{1}, \cdots, K_{m-1}\right)^{T}$, and $\xi=\left(\xi_{0}, \xi_{1}, \cdots, \xi_{m-1}\right)^{T}$. The coefficients of $\mathcal{P}$ are given by $\mathcal{P}_{i, j}=\left(\mathbf{q}_{j}^{\prime \perp}, \mathbf{q}_{i}^{\prime}\right)_{E}$.

\section{SUBSONIC CASE}

In this section, the sound generated by a fixed vortex-pairing event in a subsonic mixing layer is investigated. First, results from direct computations are presented. Then a linear global-mode analysis in conducted. A non-linear Koopman-mode analysis is finally used to reconstruct both the aerodynamic and acoustic fields.

\section{A. Direct computations}

\section{Configuration and numerical specifications}

The first case considered is a cold subsonic Mach 0.5/0.25 mixing layer at a Reynolds number $R e=\rho_{\infty} U_{c} \delta_{\omega}(0) / \mu_{\infty}=1500$, based on the initial vorticity thickness $\delta_{\omega}(0)$. The reference velocity is $U_{c}=\left(U_{1}+U_{2}\right) / 2$ where $U_{1}=0.5 c_{\infty}$ and $U_{2}=0.25 c_{\infty}$ are the speeds in the 
Global and Koopman modes analysis of sound generation in mixing layers

upper and lower streams, respectively. The thermodynamic variables are the same in both streams with $T_{\infty}=298 \mathrm{~K}, p_{\infty}=101300 \mathrm{~Pa}, \rho_{\infty}=p_{\infty} /\left(r T_{\infty}\right), c_{\infty}=\sqrt{\gamma r T_{\infty}}$, and $\mu_{\infty}=\mu\left(T_{\infty}\right)$.

The computational domain has dimensions $\left(L_{x}, L_{y}\right)=(275, \pm 235) \delta_{\omega}(0)$, including the sponge zone. The mesh is built up from a nonuniform Cartesian grid of 700 by 382 grid points in the $x$ - and $y$-directions, respectively. The grid along $x$ is uniform with spacing $\Delta x_{\min }=0.32 \delta_{\omega}(0)$ up to $x=195 \delta_{\omega}(0)$. The grid is then progressively stretched from $x=195 \delta_{\omega}(0)$ to $x=275 \delta_{\omega}(0)$ to form the sponge zone as defined in section II A. Along the normal direction, the grid is stretched at a rate of $1.8 \%$ symmetrically from $y=0$ to $y= \pm 90 \delta_{\omega}(0)$, with $\Delta y_{\min }=0.16 \delta_{\omega}(0)$ at $y=0$. The step size is then kept constant up to $\pm 235 \delta_{\omega}(0)$.

\section{Base flow}

First a base flow is obtained from the solution of the full Navier-Stokes equations (1) without inlet perturbations. The initial flow is a parallel hyperbolic-tangent profile for the streamwise velocity:

$$
\frac{u_{\text {ini }}(y)}{U_{c}}=1+R \tanh \left(\frac{2 y}{\delta_{\omega}(0)}\right)
$$

where $R=\left(U_{1}-U_{2}\right) /\left(U_{1}+U_{2}\right)$ is the velocity ratio, equal to $1 / 3$ in the present case. The mean convective Mach number is $M_{c}=U_{c} / c_{\infty}=0.375$. The temperature is initialized using the Crocco-Busemann relationship:

$$
T_{i n i}(y)=T_{\infty}+\frac{1}{2 c_{p}}\left(u_{i n i}(y)-U_{2}\right)\left(U_{1}-u_{i n i}(y)\right)
$$

In the absence of inlet perturbations, a steady solution can be reached due to the convective nature of the instabilities. After a transient period, the flow is seen to relax toward a steady state as depicted in Figure 1(a). 600000 iterations are necessary to saturate residual values.

The base flow spreads slowly along the streamwise direction due to the external flow entrainment and the viscous diffusion. The streamwise velocity profile is very close to the hyperbolic-tangent function (15), as shown in Figure 1(b). Note that several methods may have been used to compute a base flow. For instance, Lesshafft et al. ${ }^{28,29}$ used a solution of the steady compressible boundary layer equations. The advantage in the present study is that the base flow is a solution of the governing equations in the discrete sense. 
(a)

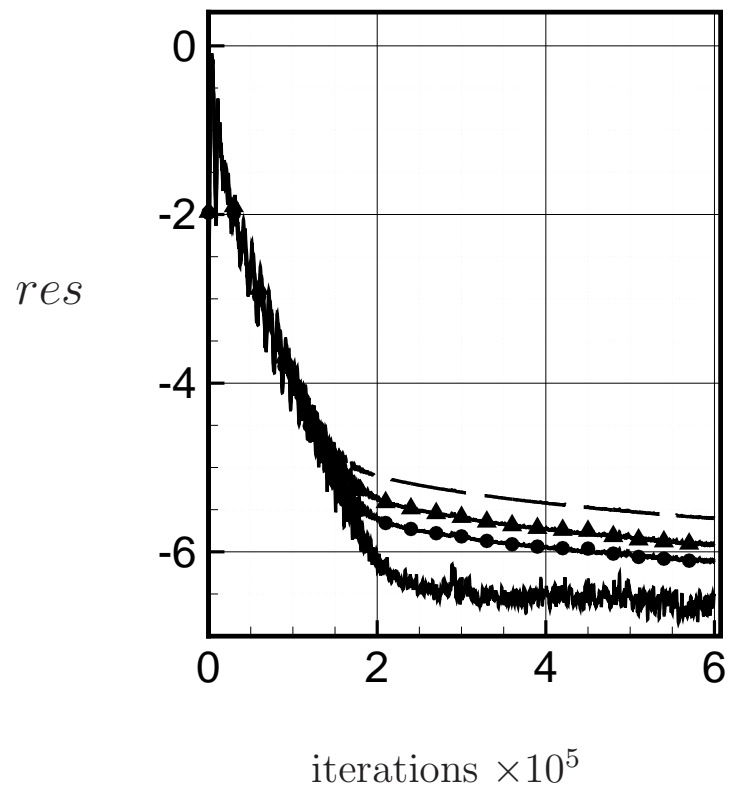

(b)

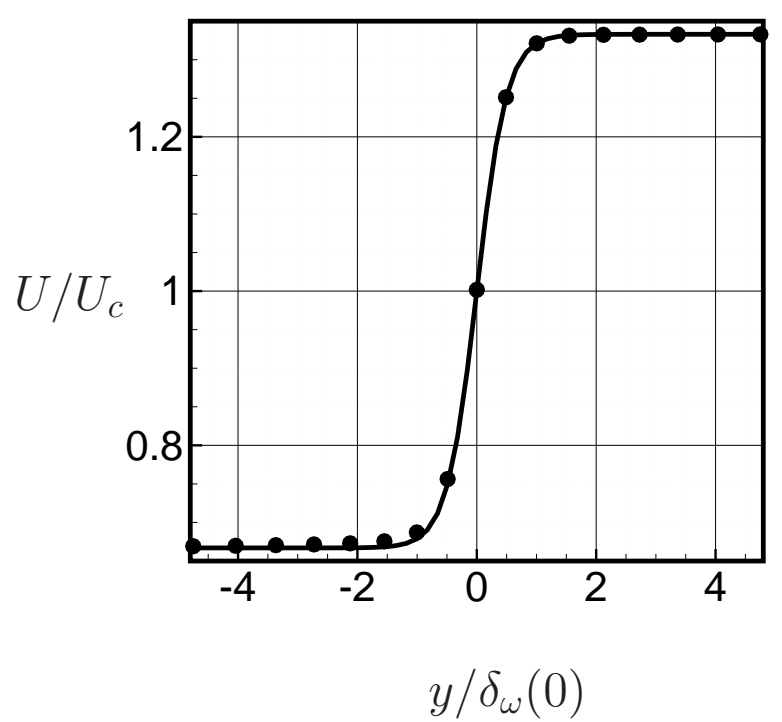

FIG. 1. Base flow for the subsonic mixing layer: (a) convergence of normalized residuals (in logarithmic scale) for the conservative variables as a function of time iterations: $-\quad-\quad \rho,-\bullet \rho u$,

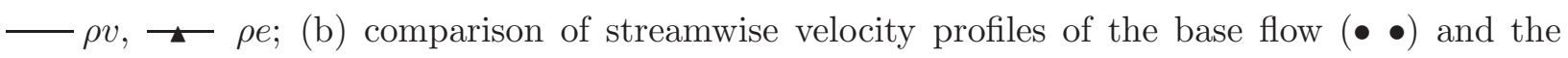
hyperbolic-tangent function (15) (- ) at the inlet plane.

\section{NLDE results}

The local linear stability theory allows us to determine the fundamental frequency $f_{0}$ at the inlet $f_{0}=\omega / 2 \pi$ with $\omega$ the circular frequency $\approx 2 \pi \times 0.82 U_{c} / \delta_{\omega}(0)$ (see Appendix A). To fix the vortex pairing location, the flow is forced artificially at its fundamental frequency $f_{0}$ and its first sub-harmonic $f_{0} / 2$ as in Bogey et al. ${ }^{7}$ and Colonius et al. ${ }^{10}$. The eigenfunctions and corresponding streamwise wavenumbers obtained from the local stability theory are integrated in the inflow boundary condition (6). This forcing condition is applied at every time step. The NLDE equations are run for 100000 iterations with a timestep $\delta t U_{c} / \delta_{\omega}(0) \simeq 0.039$.

The results from the NLDE solver (§II A 2) using the disturbance equations about the base flow are compared to the solution of the non-perturbative equations ( $(I I A 1)$ in Figures 2 and 3 at the same instant. The similarities are evident for both the near-field vortical flow and the far-field acoustic field, validating the use of the NLDE with a base flow. The 
Global and Koopman modes analysis of sound generation in mixing layers

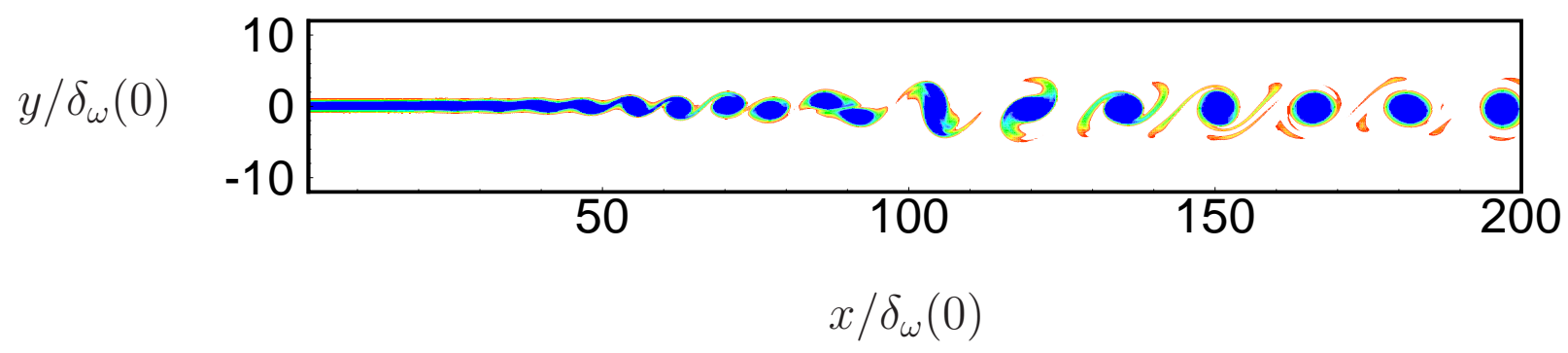

(a)

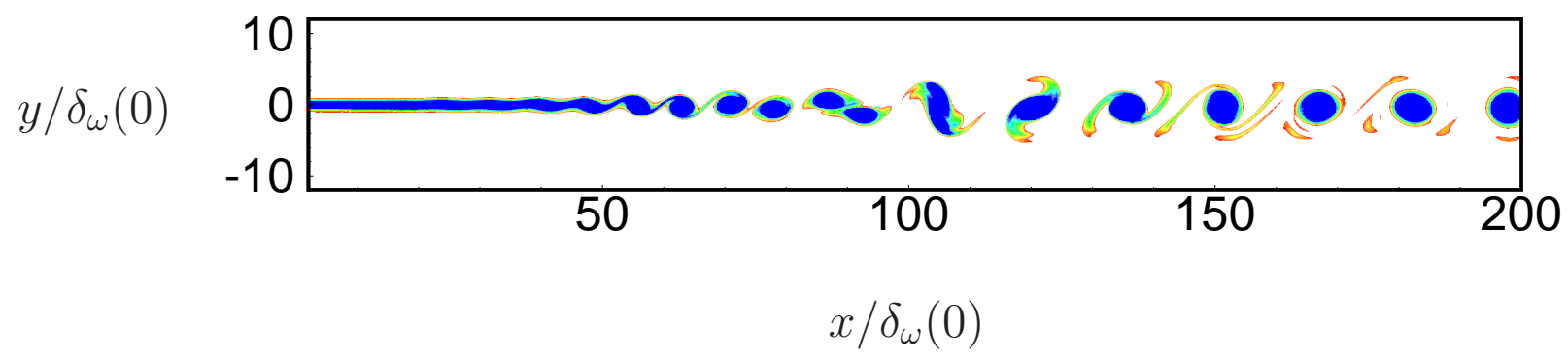

(b)

FIG. 2. Direct computations for the subsonic case. The snapshots of the total spanwise vorticity show the vortex pairing process: (a) full Navier-Stokes simulation; (b) NLDE simulation. Contours levels range from -0.20 to -0.05 where the values are made dimensionless by $U_{c}$ and $\delta_{\omega}(0)$.

spanwise vorticity plot in Figure 2 exhibits the saturated Kelvin-Helmholtz rolls and the fixed vortex pairings around $x \simeq 100 \delta_{\omega}(0)$. The acoustic pattern in Figure 3 illustrates the rotating quadrupolar radiation ${ }^{7}$ resulting from vortex-sound generation mechanism. The principal directions of the wavefronts depend on convection effects in the upper and lower streams.

The directivity of the wave radiation provides a quantitative knowledge of noise emissions. To this aim, the sound pressure level

$$
S P L=20 \log _{10}\left(\frac{p_{r m s}^{\prime}}{p_{r e f}^{\prime}}\right)
$$

where $p_{r e f}^{\prime}=2 \times 10^{-5} \mathrm{~Pa}$ is the reference pressure and $p_{r m s}^{\prime}$ is the root mean square (rms) value is computed during one pairing period. The directivity is then evaluated by interpolating the values of $p_{r m s}^{\prime}$ on an arc of radius $100 \delta_{\omega}(0)$ centered at the apparent source location $(x, y)=\left(100 \delta_{\omega}(0), 0\right)$. This apparent source location corresponds to the vortex pairing zone 
Global and Koopman modes analysis of sound generation in mixing layers

(a)

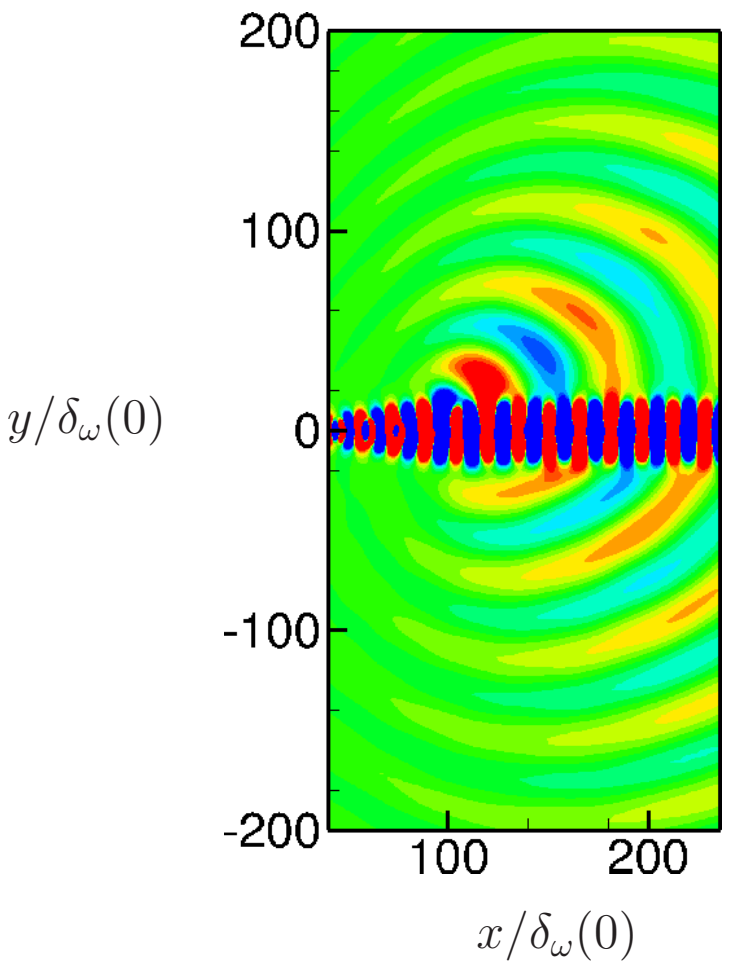

(b)

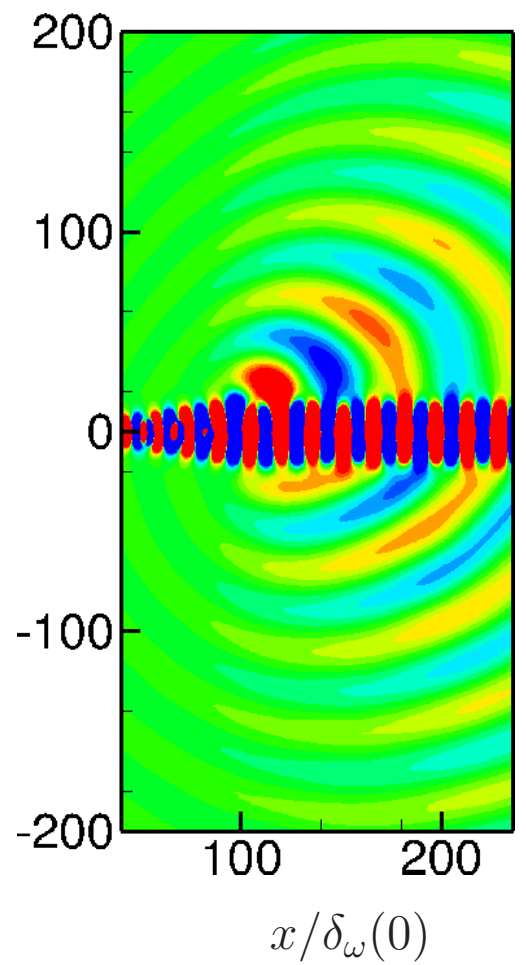

FIG. 3. Direct computations for the subsonic case. The fluctuating pressure over the entire domain shows the far-field noise radiation: (a) full Navier-Stokes simulation; (b) NLDE simulation. The pressure is made dimensionless with $\rho_{\infty} c_{\infty}^{2}$. Contours levels range from $-3 \times 10^{-5}$ to $3 \times 10^{-5}$.

which is responsible for the acoustic radiation ${ }^{7}$. The directivity is shown in Figure 4(a). For the upper stream, the maximum of acoustic radiation appears at $\theta \approx 38^{\circ}$ measured from the streamwise direction. A smaller angle $\theta \approx-21^{\circ}$ from the axis is noticed for the emission lobe in the lower stream.

Lastly, the frequency content in the flow is characterized through the evolution of the modal energy as defined by (11) but integrated only in a normal section $(d V \equiv d y)$, plotted in Figure 4(b). The excited modes at frequencies $f_{0} / 2$ and $f_{0}$ fisrt undergo an exponential growth, in good agreement with the local stability prediction in Appendix A, underlying the almost parallel character of the base flow. The fundamental mode saturates first and is then overwhelmed by the subharmonic component. This modification of the dominant frequency marks the location of the pairing event. Higher-order modes such as $3 f_{0} / 2$ and $2 f_{0}$ are then triggered through nonlinear interactions. 
(a)

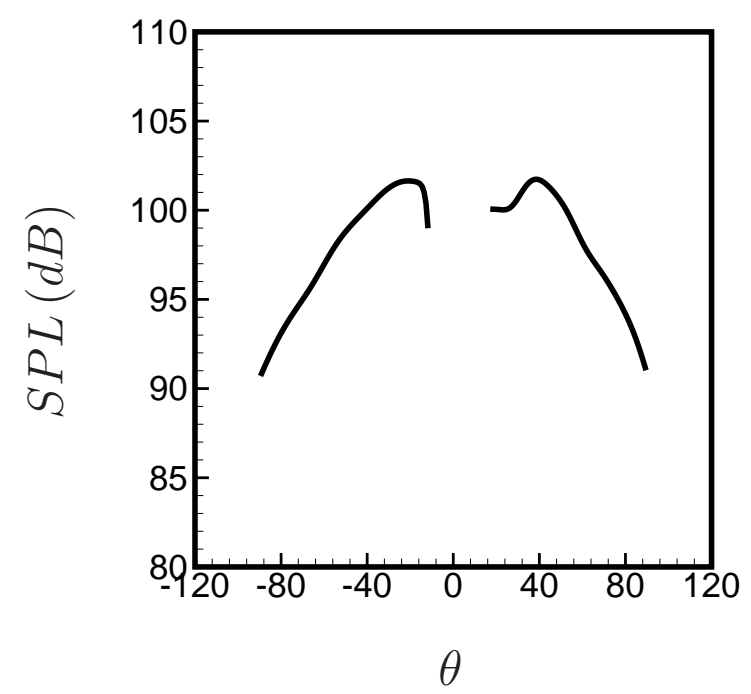

(b)

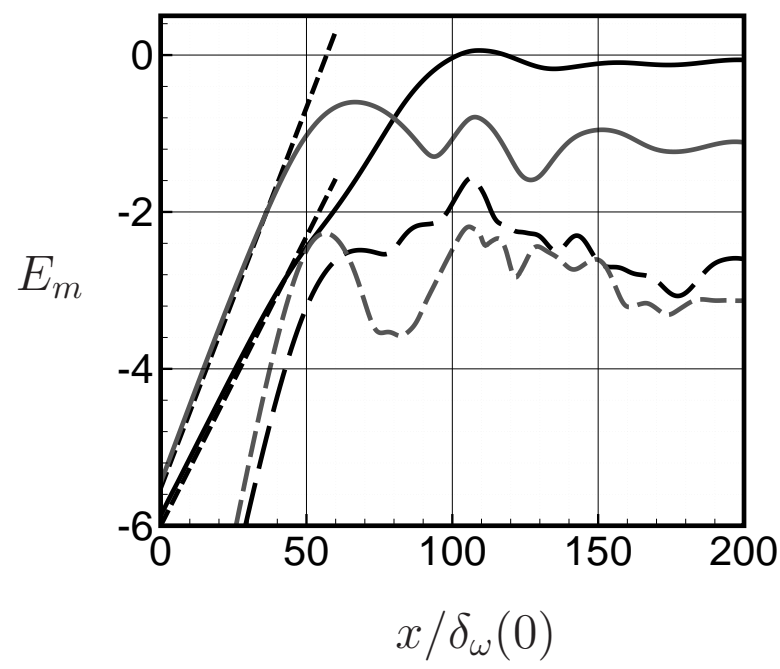

FIG. 4. NLDE computation for the subsonic case: (a) Far-field directivity for an arc of radius $100 \delta_{w}(0)$ centered at $x=100 \delta_{\omega}(0)$; (b) Integrated modal energy for $f_{0} / 2(-), f_{0}(-), 3 f_{0} / 2$

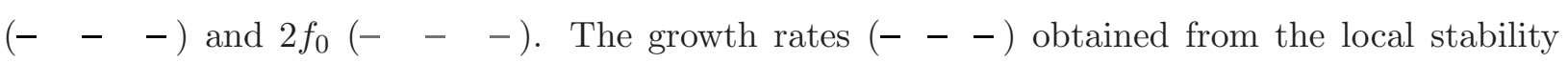
analysis in Appendix $\mathrm{A}$ for $f_{0} / 2$ and $f_{0}$ are shown for comparison.

\section{B. Linear analysis: global modes and LDE simulation}

\section{Global modes: numerical parameters and spectrum}

In this section, the nonlinear terms in (4) are omitted and the equations are referred to as linear disturbance equations (LDE). The sequence of snapshots is initiated by a localized impulse in the mixing layer. Even with a divergence-free initial velocity field, it is important to specify properly the thermodynamic variables $p^{\prime}$ and $\rho^{\prime 20,37}$ to avoid spurious noise. Hence, we use as an initial condition a Taylor's vortex with a given amplitude $A=10^{-6}$ :

$$
\begin{aligned}
& u^{\prime}=A\left(y-y_{0}\right) e^{\Lambda R^{2}}, v^{\prime}=-A\left(x-x_{0}\right) e^{\Lambda R^{2}} \\
& \text { where } R=\sqrt{\left(x-x_{0}\right)^{2}+\left(y-y_{0}\right)^{2}} \text { and } \Lambda=-\ln \left(2 / b^{2}\right)
\end{aligned}
$$

The Gaussian half-width $b$ is taken as $3 \Delta y_{\min }$ and the vortex is centered at $\left(x_{0}, y_{0}\right)=$ $\left(20 \delta_{\omega}(0), 0\right)$. The initial pressure is known analytically:

$$
p^{\prime}=-\rho_{\infty} \frac{A^{2}}{4 \Lambda} e^{2 \Lambda R^{2}}
$$


Global and Koopman modes analysis of sound generation in mixing layers

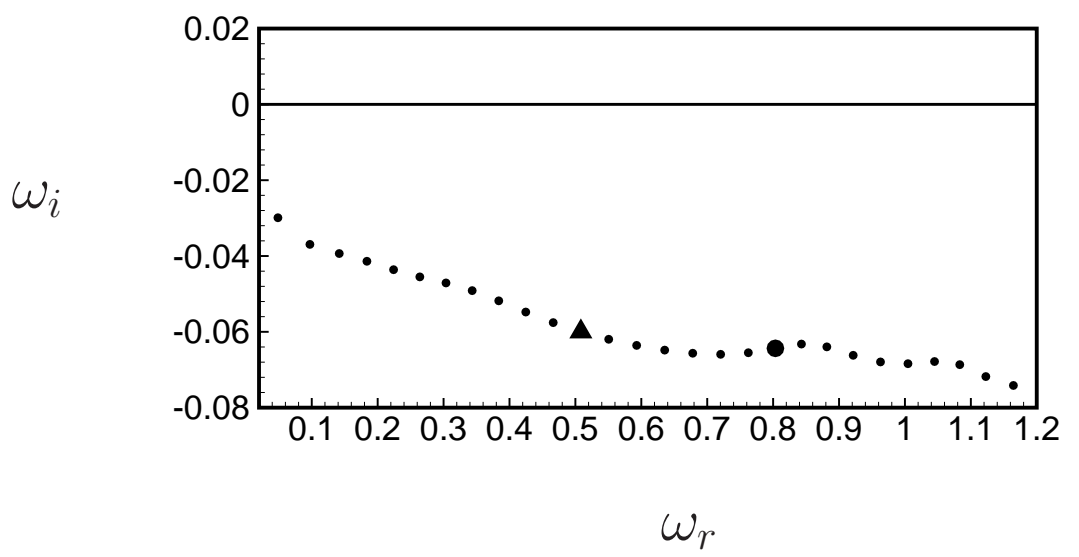

FIG. 5. Global spectrum of the subsonic mixing layer is shown. The eigenvalues are dimensionless by $\delta_{\omega}(0)$ and $U_{c}$.

As disturbances grow in amplitude and propagate downstream, the sequence of snapshots separated by a sampling period $\Delta t$ is carried out. The sampling period in the present investigation is fixed to a value which guarantees a resolution up to $2 f_{0}$ (Nyquist criterion). The snapshots are realized for a window extending from $x=0$ to $x=115 \delta_{\omega}(0)$ and $y=$ $\pm 45 \delta_{\omega}(0)$. In the following, $N=240$ snapshots are used to extract the dominant global modes. The number of snapshots are chosen to satisfy a minimal residual value for the Arnoldi algorithm. In addition, one may precise that the base flow is slighty nonparallel which prevents ill-conditioned operator ${ }^{11}$.

The global spectrum is depicted in Figure 5. One may observe that all the global modes are damped temporally, illustrating the well-known noise amplifier behaviour of a coflow mixing layer. Figure 6 shows two samples of eigenvectors at two different circular frequencies. The wavelength is seen to decrease as the circular frequency increases and a large spatial amplification is observed for both frequencies. These remarks are consistent with the convective nature of instabilities arising in the mixing layer.

By plotting the pressure component of global modes no acoustic radiation originating from the shear layer is visible in contrast with the NLDE simulation. To illustrate this last comment, the relative phase velocities of the two global modes displayed in Figure 6 are computed for the lower and upper streams. For that purpose, we use a weakly nonparallel assumption to derive an expression of the wavenumber $\alpha_{r}$ along the streamwise direction. 
Global and Koopman modes analysis of sound generation in mixing layers

(a)

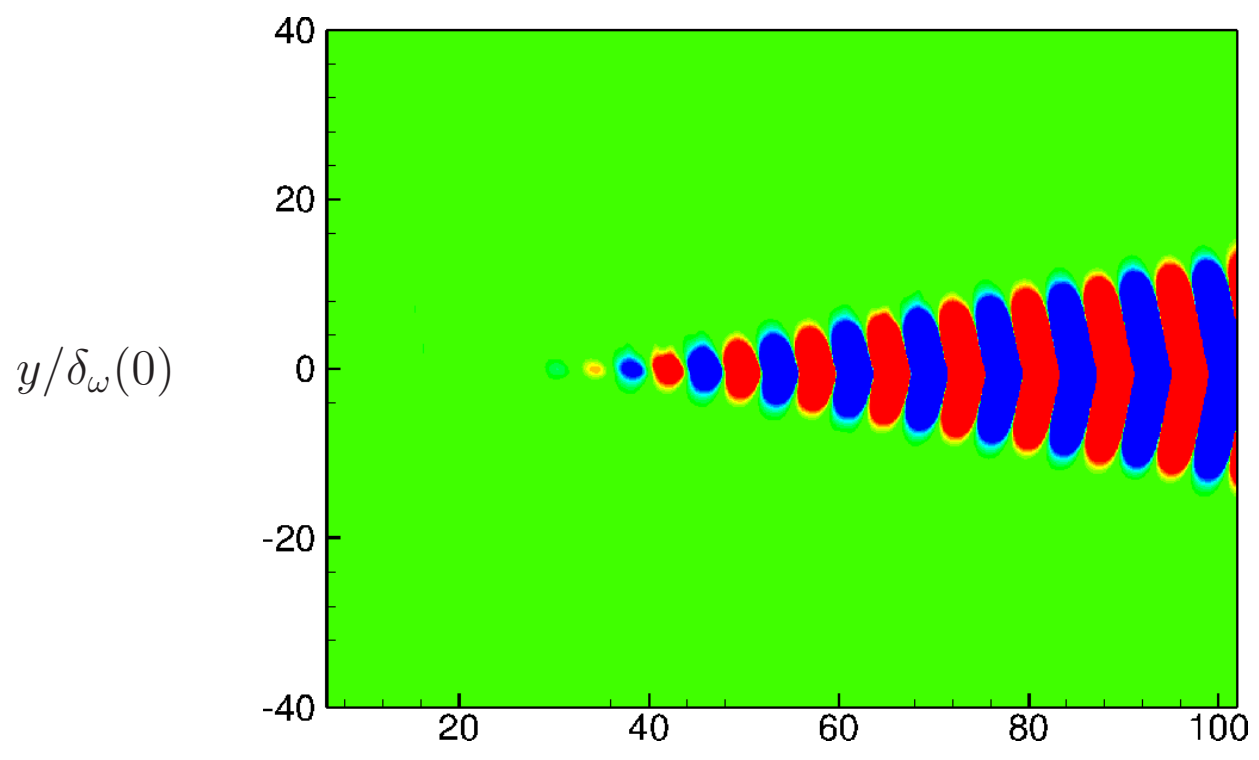

(b)

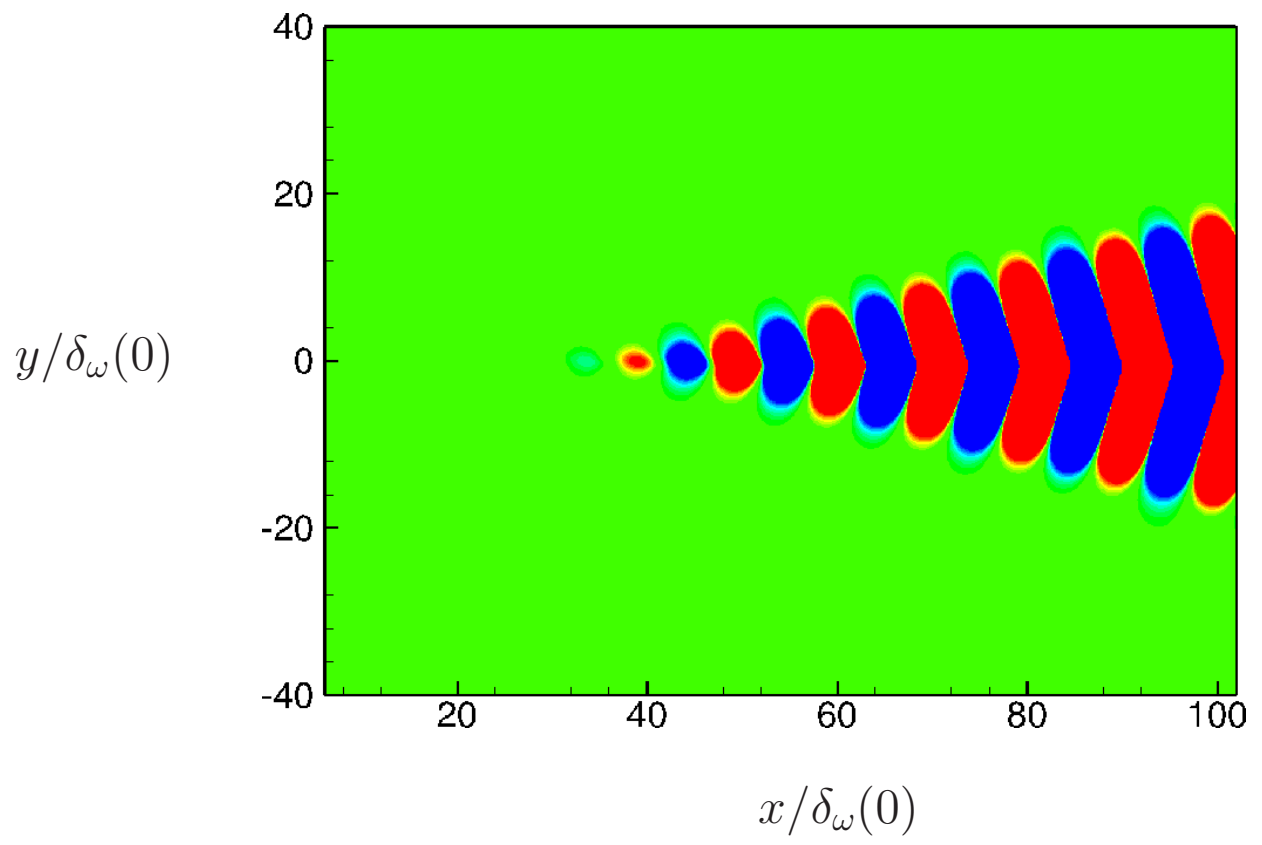

FIG. 6. Pressure fluctuations associated with eigenvalues marked by (a) $\bullet$ and (b) $\Delta$ in Figure 5.

Hence, (20) is evaluated at the maximum value of $|\hat{v}|$ along $x$ as

$$
\alpha_{r}=\frac{\partial}{\partial x}\left(\tan ^{-1} \frac{\hat{v}_{i}}{\hat{v}_{r}}\right)
$$

where $\bullet_{r}$ and $\bullet_{i}$ denote the real and imaginary parts, respectively. The relative phase velocity 
Global and Koopman modes analysis of sound generation in mixing layers

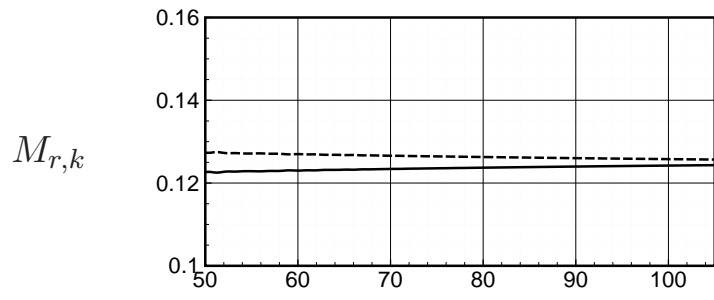

$$
x / \delta_{\omega}(0)
$$

(a)

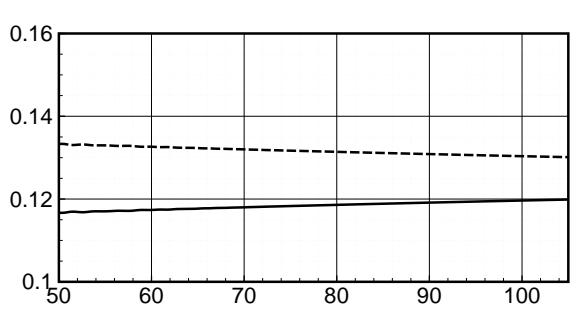

$x / \delta_{\omega}(0)$

FIG. 7. Phase speeds $M_{r, 1}(-)$ and $M_{r, 2}(-\quad-\quad)$ corresponding to the eigenvalues marked by: (a) $\triangle$ and (b) • in the spectrum of Figure 5 .

is defined as

$$
M_{r, k}=\frac{\left|\omega_{r} / \alpha_{r}-U_{k}\right|}{c_{\infty}}
$$

where $k=1,2$ for the upper and the lower stream respectively and $c_{\infty}$ denotes the speed of sound. The relative phase velocities of the global modes of Figure 6 are plotted in Figure 7 for the lower and upper streams. As expected, each global mode has a subsonic phase velocity relative to the ambient sound speed. Therefore, these instability waves can not generate sound ${ }^{47}$. A similar behaviour is observed for all global modes of the spectrum shown in Figure 5.

\section{Response to a harmonic forcing and noise emissions}

Lastly, the method of artifical excitation with real frequency is classically adopted to understand the noise generation process in open flows. For instance, the subsonic mixing layer is forced at the fundamental and subharmonic frequencies in the NLDE simulation, presented in the section III A 3, to get insight into noise emissions. Since the pioneering work of Cossu \& Chomaz $^{11}$, it is well established that temporally damped global modes may lead to transient growth. To characterize this growth, the transfer function associated with the response to a harmonic forcing may be investigated ${ }^{42}$. Note that the harmonic forcing is based on an appropriate superposition of the previously computed global modes. Since each global mode is individually not radiating, a similar behaviour is expected for the summation of modes. 
Global and Koopman modes analysis of sound generation in mixing layers

To model the external excitation, a small-amplitude forcing term $\mathbf{f}=\hat{\mathbf{f}} e^{-i \omega_{f} t}$ is added to the linear system, characterized by its angular frequency $\omega_{f}$ and its spatial structure $\hat{\mathbf{f}}$. The flow being globally stable, the asymptotic response is governed by

$$
\mathbf{q}^{\prime}=\sum_{k=0}^{m-1} K_{k} \hat{\mathbf{q}}_{k} e^{-i \omega_{f} t} \quad \text { with } \quad K_{k}=\frac{K_{k}^{f}}{i \omega_{f}-i \omega_{k}}
$$

where the expansion coefficients of $\hat{\mathbf{f}}$ into a global modes basis have been noted $K_{k}^{f}$. To measure the response of our linear system to a harmonic forcing at a real frequency $\omega_{f}$, we introduce the following quantity:

$$
R\left(\omega_{f}\right)=\frac{\left\|\mathbf{q}^{\prime}\right\|^{2}}{\|\hat{\mathbf{f}}\|^{2}}
$$

In practice, the forcing term takes the form of a wavepacket centered near $x / \delta_{\omega}(0)=40$ derived from an instantaneous snapshot of the LDE simulation, which was used in the previous section to extract the global modes. An orthogonal projection is employed to recover the expansion coefficients. A total of $m=62$ global modes are considered, including positive and negative circular frequencies. The transfer function is illustrated in Figure 8(a). We observe a broadband hump centered around a dimensionless forcing frequency $\omega_{f} \approx 0.67$, which is somewhat lower than the fundamental frequency as computed by a local stability theory at the inlet (see Appendix A). This shift may be attributed to the thickening of the shear layer along the streamwise direction for the base flow.

The fluctuating pressure associated with the asymptotic response is depicted for the subharmonic in Figure 8(b). As expected from the analysis of individual global modes, no acoustic radiation is observed for the global response at $f_{0} / 2$. Similar observation could be made for all forcing frequencies since the present study is restricted to the linear regime. Hence, this analysis illustrates the ability of the global modes to evaluate the linear amplification of perturbations in subsonic mixing layer but also exhibits its failure to represent the noise radiation observed in the NLDE simulation.

\section{Comparison between $L D E$ and NLDE simulations}

To further illustrate the conclusions given by our global modes analysis, a LDE simulation is performed by considering the forcing condition detailed in section III.A.3. The result is displayed in Figure 9. From the pressure field, in agreement with the global modes analysis, 
(a)

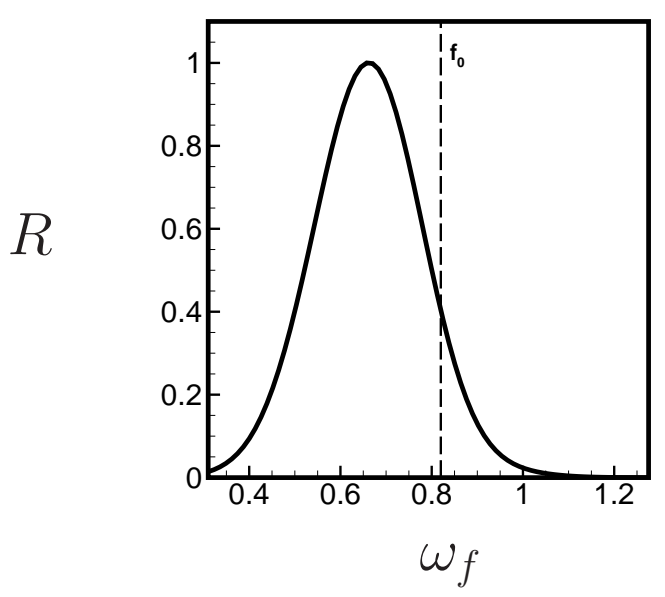

(b)

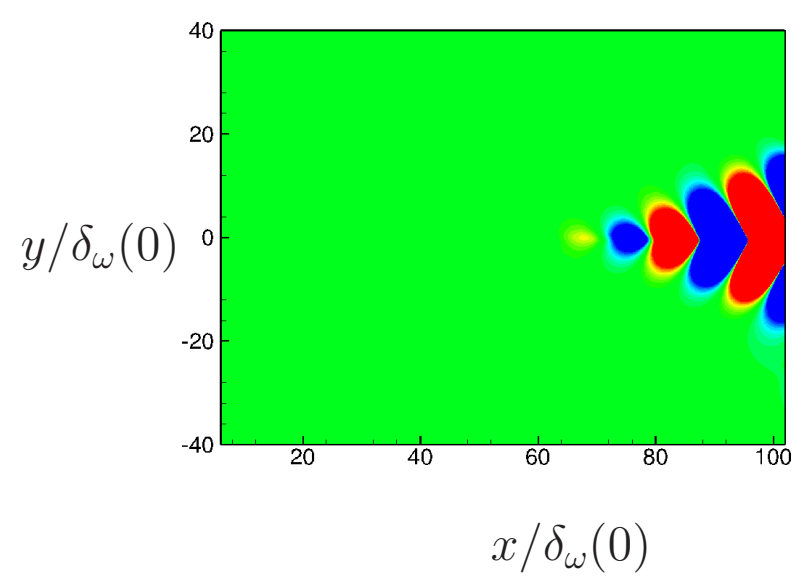

FIG. 8. Linear response to the forcing term based on 62 global modes: (a) Transfer function normalized by its maximum value. The dimensionless fundamental circular frequency at the inlet is referenced with a vertical dashed line; (b) Spatial structure associated with the response at $f_{0} / 2$.

no acoustic radiation appears. In addition, the influence of the nonlinear terms is characterized through the evolution of the integrated energy in a normal section for disturbances, at a given time. In particular, a significant gap between the LDE and the NLDE simulations is observed at the pairing location giving strong evidence that the nonlinear terms are not small enough to be neglected.

\section{Nonlinear model}

In this section, the subsonic mixing layer is used to illustrate the possibility to extract the coherent structures responsible for the radiated noise in a nonlinear framework, from a DMD analysis.

\section{DMD analysis: Koopman modes}

For that purpose, the DMD analysis is applied to the NLDE database, described in section III A, over the entire domain, referred to as $\mathcal{D}_{f}$, and over a subdomain, noted $\mathcal{D}_{s}$, extending from $x=0$ to $250 \delta_{\omega}(0)$ and from $y=-15 \delta_{\omega}(0)$ to $y=15 \delta_{\omega}(0)$. This subdomain is thus dedicated to the analysis of the vortical structures in the source region. A number 


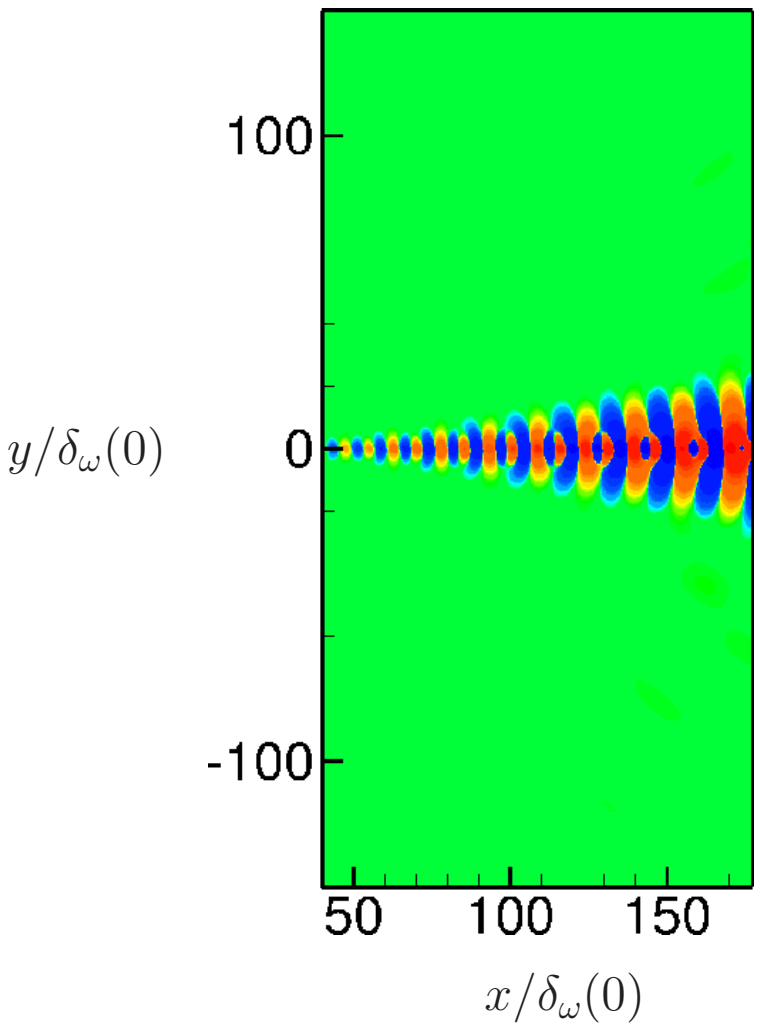

(b)

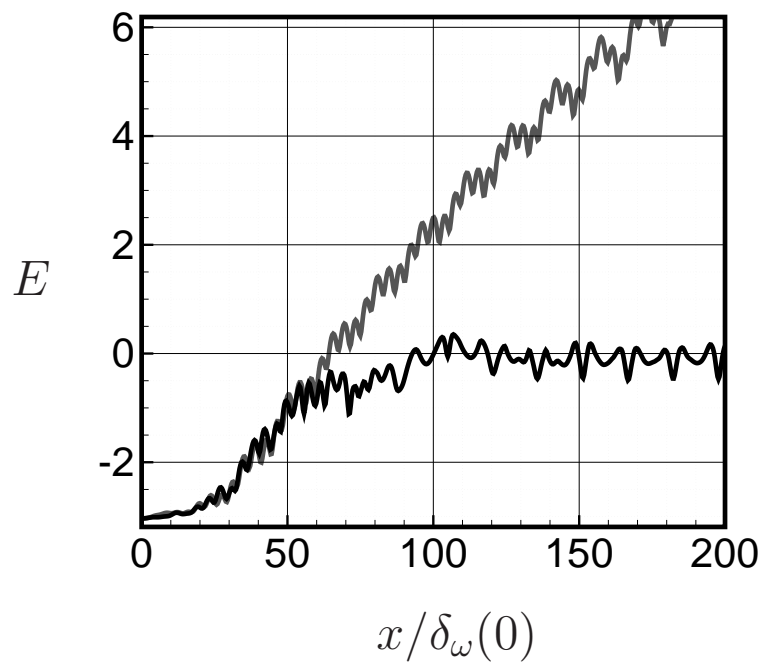

(a)

FIG. 9. LDE simulation for the subsonic case; (a) the pressure field is shown; (b) integrated energy associated with disturbances for $\operatorname{NLDE}(-)$ and $\operatorname{LDE}(-)$ simulations are plotted in a logarithmic scale.

of $N=210$ instantaneous snapshots of the density, velocity and pressure fluctuations are recorded at a sampling rate $\Delta t U_{c} / \delta_{\omega}(0)=0.6358$, which corresponds approximately to 12 points per period at the fundamental frequency $f_{0}$.

The eigenvalues of the Koopman operator associated with $\mathcal{D}_{f}$ and $\mathcal{D}_{s}$ are shown in Figure 10(b) and 11(b), respectively. Nearly all the Ritz values are found to lie on the unit circle $\left|\lambda_{j}\right|=1$, which means that the sample points lie on or near an attracting $\operatorname{set}^{38}$. Figures 10(a) and 11(a) display the normalized energies of the extracted modes as a function of the frequency. Each mode represented by its norm is depicted with a vertical line and appear as a complex conjugate pair. The two dominant peak frequencies containing the highest energy are then identified and marked with the same colours as the corresponding eigenvalues in Figures 10(b) and 11(b). These two modes are associated with the first subharmonic $f_{0} / 2$ and the fundamental mode $f_{0}$. Higher frequencies are also excited, corresponding to sum 
Global and Koopman modes analysis of sound generation in mixing layers

(a)

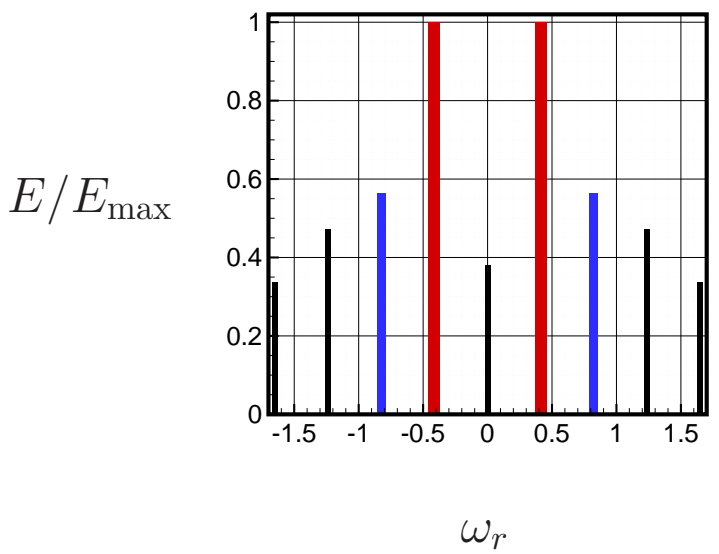

(b)

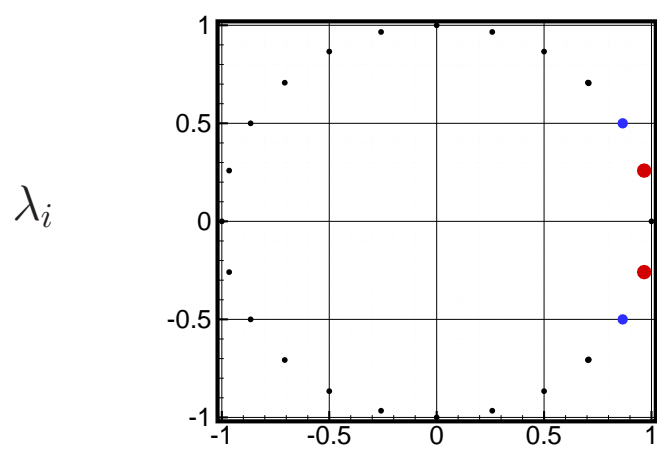

$\lambda_{r}$

FIG. 10. DMD analysis performed over the entire computational domain $\mathcal{D}_{f}$ : (a) Energy spectrum; (b) Ritz values. The values are made dimensionless by $\delta_{\omega}(0)$ and $U_{c}$.

(a)

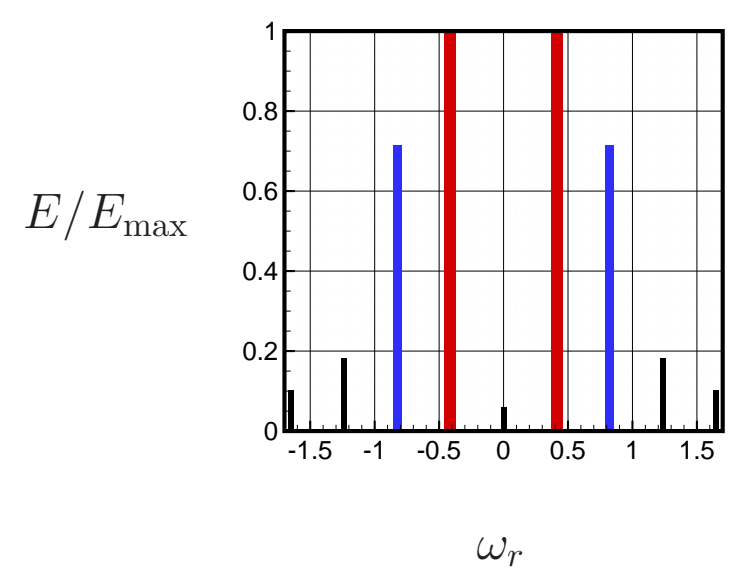

(b)

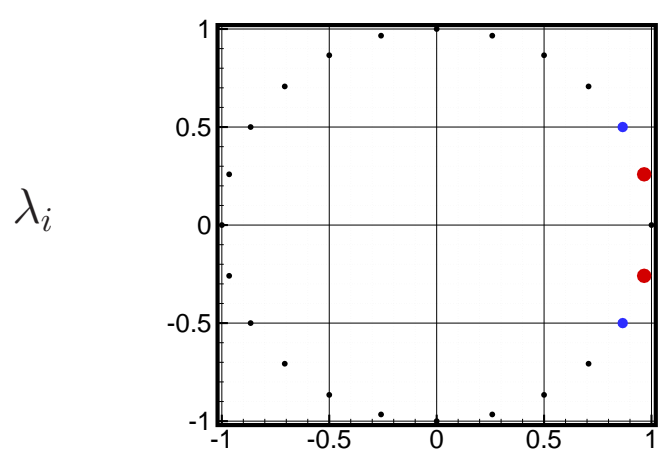

$\lambda_{r}$

FIG. 11. DMD analysis performed over the subdomain $\mathcal{D}_{s}$ : (a) Energy spectrum; (b) Ritz values. The values are made dimensionless by $\delta_{\omega}(0)$ and $U_{c}$.

and difference of the frequencies. For instance, the nonlinear interaction of $f_{0} / 2$ and $f_{0}$ results in a peak at $3 f_{0} / 2$, and so on. One may notice that a few modes dominate the energy spectrum and that the energy distribution among the Koopman modes is roughly similar for $\mathcal{D}_{f}$ and $\mathcal{D}_{s}$. 
Global and Koopman modes analysis of sound generation in mixing layers

To represent the vorticity and pressure modes associated with each energy peak, the Koopman modes are projected onto an instantaneous snapshot from the NLDE simulation. The pressure fluctuation components are displayed in Figure 12 for the modes at $f_{0}$ and $f_{0} / 2$ from the analysis on $\mathcal{D}_{f}$. The acoustic waves at $f_{0} / 2$ emanate from the region where the pairings occur. Besides, the mode $f_{0}$ produces a weak far-field noise compared to the mode at $f_{0} / 2$. Similar observations could also be made for $3 f_{0} / 2$ or $2 f_{0}$. Therefore, the first subharmonic mode contributes mainly to the acoustic radiation in the mixing layer. Very different results would be obtained with a proper orthogonal decomposition (POD). For instance, Druault et al. ${ }^{14}$ show that the most energetic POD modes are not associated with the main noise radiation, which can be obtained from a series of higher-order POD modes. As underlined by Schmid ${ }^{41}$, higher-order POD modes, corresponding to a smaller energetic content, can still play a significant dynamic role within the snapshot sequence.

In addition, the acoustic behaviour observed in Figure 12 gives some insight about the slight modification of the relative magnitudes of Koopman modes for $\mathcal{D}_{f}$ and $\mathcal{D}_{s}$ as depicted in Figures 10 (a) and 11 (a). As discussed above, the noise is mainly produce by the subharmonic $f_{0} / 2$. As a consequence, a larger contribution of the pressure component with respect to the inner product (11) is expected for $f_{0} / 2$ by considering $\mathcal{D}_{f}$ which includes the radiated noise. This additional contribution is not captured by $\mathcal{D}_{s}$ where the DMD analysis is restricted to the vortical structures in the near field. For instance, the ratio between the energy peaks associated with $f_{0}$ and $f_{0} / 2$ is $\approx 0.72$ for $\mathcal{D}_{s}$ and $\approx 0.56$ for $\mathcal{D}_{f}$. Finally, it is worth noting from the Koopman modes magnitudes that the zero frequency mode plays a significant role in the dynamics with a magnitude of the same order as $2 f_{0}$. The total vorticity, which includes the base flow, for the zero frequency mode and the time-averaged flow are shown in Figure 13 for $\mathcal{D}_{f}$. A perfect match is observed, which clearly identifies the zero frequency mode as the mean flow correction. In particular, it illustrates the nonlinear effects associated with the mixing process, with a strong modification of the total vorticity near the vortex pairing position. This nonlinear mechanism will lead to a modification of the instability waves behaviour which can not be captured with a linear theory.

Finally, to gain further insight into the revelancy of the subdomain analysis, the far-field acoustic noise is recovered via a Kirchhoff's extrapolation method from a surface enclosing the source (see Appendix B). This surface is composed of both the upper and lower boundaries of $\mathcal{D}_{s}$. For the computation of pressure fluctuations and their derivatives in (B1), we 
(a)

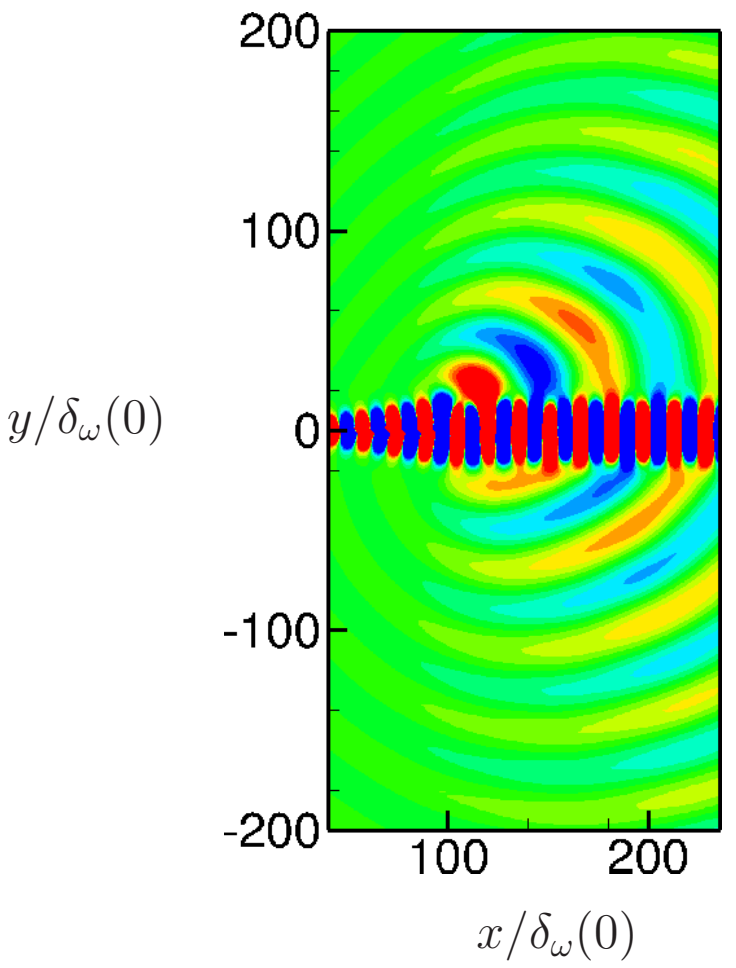

(b)

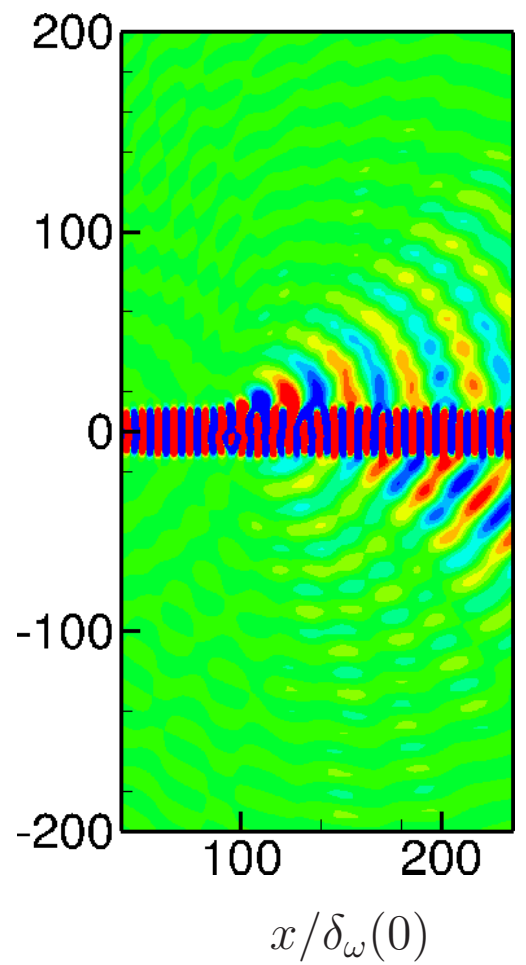

FIG. 12. Real part of the non-dimensional pressure fluctuations for Koopman modes calculated on the entire domain $\mathcal{D}_{f}$ corresponding to: (a) $f_{0} / 2$; (b) $f_{0}$. A projection on an initial snapshot is used to obtain the amplitudes. Contours levels range from $-3 \times 10^{-5}$ to $3 \times 10^{-5}$ in steps of $5 \times 10^{-6}$ in plot (a) and from $-3 \times 10^{-6}$ to $3 \times 10^{-6}$ in steps of $5 \times 10^{-7}$ in plot (b).

use the projection of the pressure field in the source region:

$$
p^{\prime}(\mathbf{x}, t)=K_{k} p_{k}(\mathbf{x}) e^{-i \omega_{k} t}
$$

with $p_{k}$ the pressure component of the corresponding Koopman mode and $K_{k}$ a weighting coefficient associated with the scalar product. As observed in Figure 14, the pressure predicted in the far field from the analysis based on $\mathcal{D}_{s}$ and formulae (24) and (B1) is in agreement with the decomposition based on the full domain $\mathcal{D}_{f}$.

In particular, the directivities associated with $f_{0} / 2$, shown in Figures 15 , indicate that the far-field radiation evaluated from $\mathcal{D}_{f}$ and $\mathcal{D}_{s}$ are of the same order of magnitude and that the directions of radiation in the upper and lower streams are in good agreement. It is worth noting that it hightlights the ability of the DMD analysis performed on the entire domain to extract accurately the dominant vortical structures together with the main contribution to the noise radiation. In addition, the Koopman modes derived from the subdomain analysis 
Global and Koopman modes analysis of sound generation in mixing layers

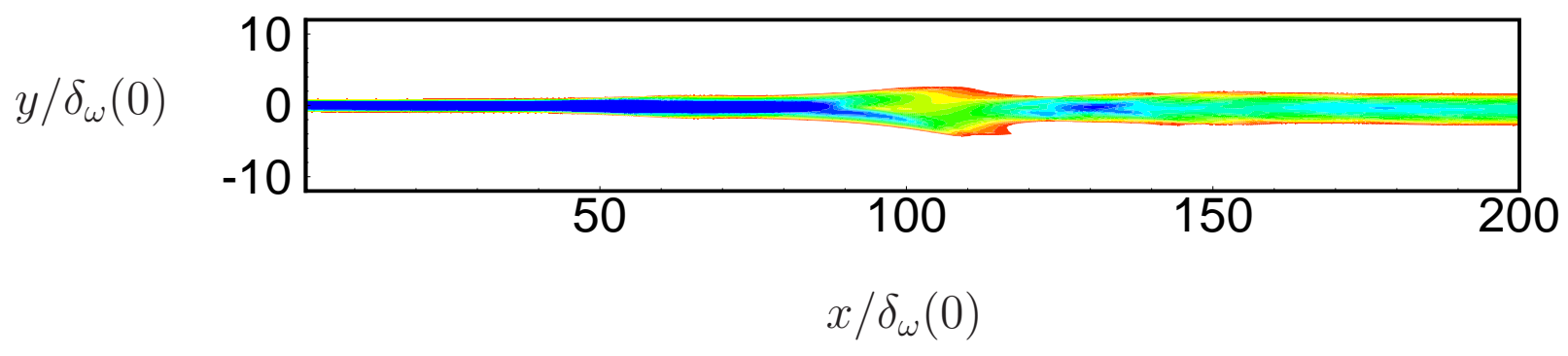

(a)

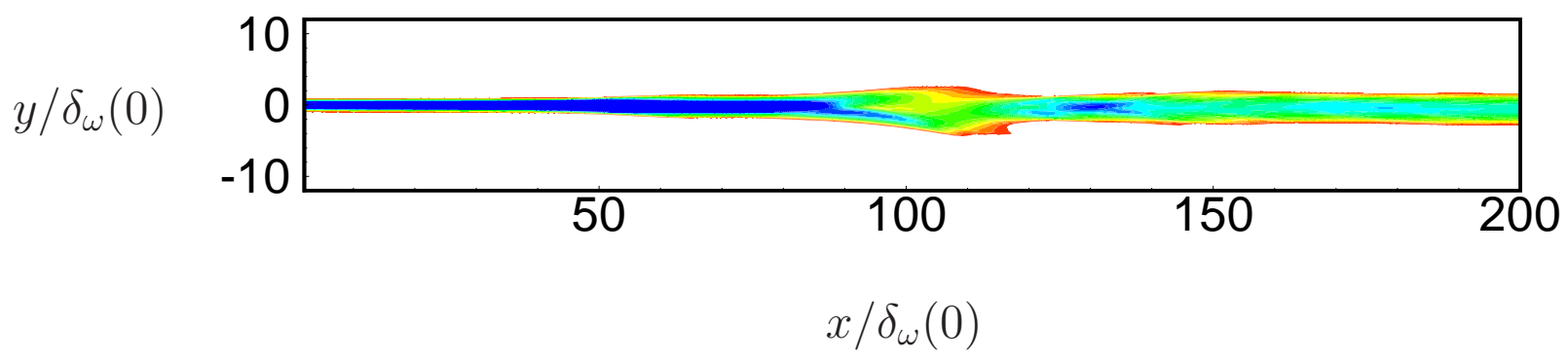

(b)

FIG. 13. The total vorticity for the zero frequency mode with respect to $\mathcal{D}_{f}$ (a) and the timeaveraged flow (b) are shown. Contours levels range from -0.20 to -0.05 .

are sufficiently accurate to be combined with a Kirchhoff's extrapolation method to recover the far-field acoustics.

We have demonstrated that the DMD analysis applied to a near-field region or to the full domain is capable of describing accurately the coherent structures which contributes mainly in terms of dynamics as well as their noise radiation. In particular, the comparison between the subdomain, corresponding to the near-field and the full domain analysis allows to distinguish the contribution of the dominant coherent structures which are involved in the hydrodynamic behaviour in the near field and their impact on the acoustic noise in the far field.

A final observation can be made: when forcing the flow with $f_{0}$ and $f_{0} / 2$, the structures associated with the Koopman modes can radiate sound directly to the far field which totally differs from the linear global modes where no radiation occurs from the global flow response to harmonic forcing at $f_{0}$ and $f_{0} / 2$. This last comment can be highlighted through the Figure 16, where cross sections of the modulus of the pressure eigenfunctions for the Koopman 
Global and Koopman modes analysis of sound generation in mixing layers

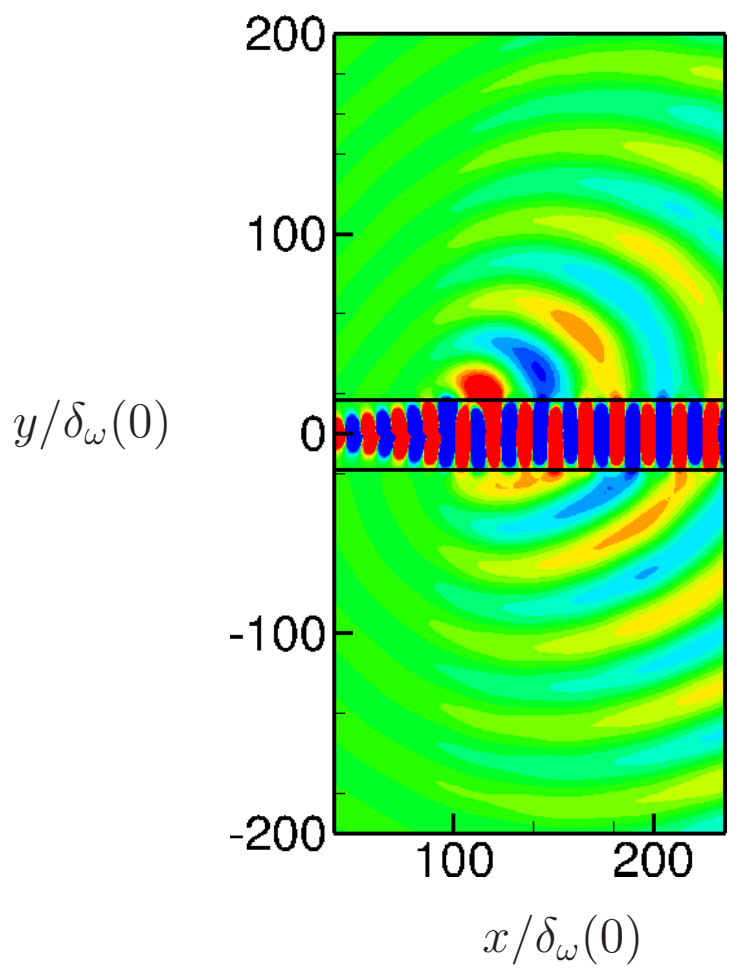

FIG. 14. Real part of the non-dimensional pressure fluctuations for Koopman modes calculated on the subdomain $\mathcal{D}_{s}$ corresponding to $f_{0} / 2$. The Kirchhoff wave extrapolation method from the boundaries of the subdomain $\mathcal{D}_{s}$ is used to determine the far-field acoustic noise. Contours levels range from $-3 \times 10^{-5}$ to $3 \times 10^{-5}$ in steps of $5 \times 10^{-6}$.

mode associated with $f_{0} / 2$ and for the global mode for $\omega_{r} \approx 0.4$ (which is closed to $f_{0} / 2$ ) at $x / \delta_{\omega}(0)=110$ are plotted. A clear exponential decay is visible for the global mode, whereas the Koopman mode reveals bounces after $|y|>30 \delta_{\omega}(0)$ which are linked to the acoustic emissions. In the subsonic case, the sound has a purely non linear origin, namely the pairing event.

Hence, being able to identify the different structures and their associated noise radiation separately, let us now consider the interactions between different eigenmodes of the Koopman operator that can potentially generate acoustic radiation. 


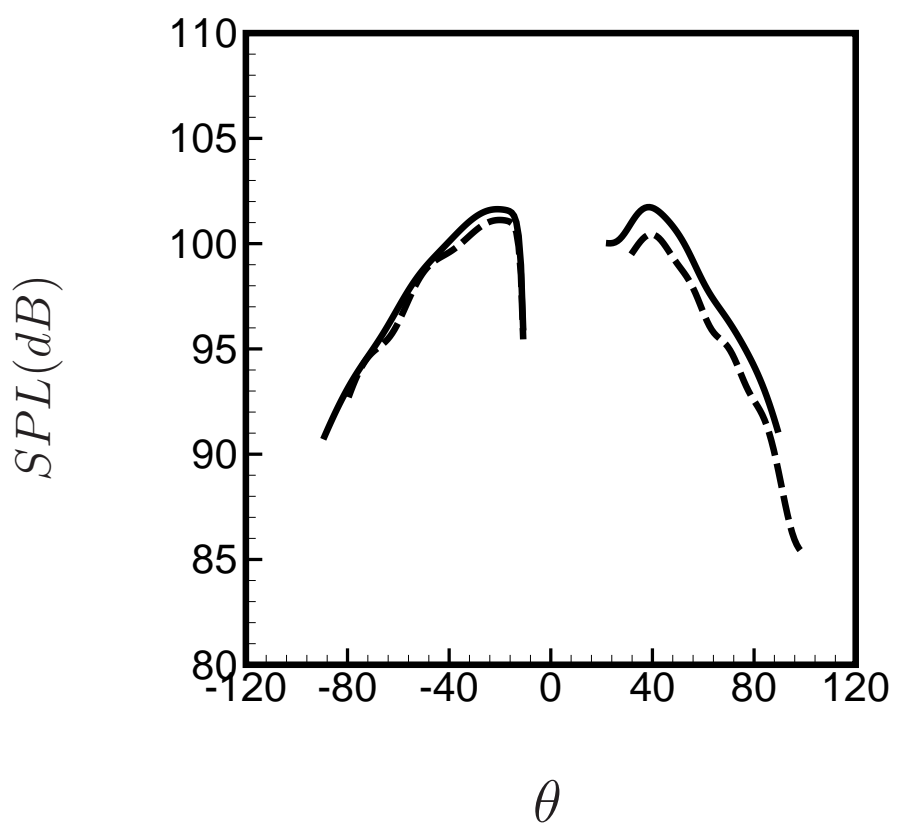

FIG. 15. Far-field directivity obtained by the DMD analysis for an arc of radius $100 \delta_{\omega}(0)$ centered at $x=100 \delta_{\omega}(0)$ for the subharmonic mode $f_{0} / 2$ : ( - ) full domain $\mathcal{D}_{f} ;\left(--{ }^{-}\right)$subdomain $\mathcal{D}_{s}$ + Kirchhoff's extrapolation method.

\section{Reconstruction of the near-and far-field dynamics}

Now, to assess the ability of the DMD analysis to reproduce both the entire shear layer dynamics in the near and far fields, a reconstruction is carried out from a selection of the dominant eigenmodes. Considering the non-orthogonality of the Koopman modes, a prior orthogonal projection onto the Koopman modes basis is used as described in the section II B 2. By increasing gradually the number of modes, the difference between an instantaneous snapshot from the NLDE simulation and the projection is made lower than a residual value of $10^{-4}$ (based on a norm associated with the scalar product (11)) for 7 modes including the zero-frequency mode. One may precise that both modes with positive frequency and their complex conjugate are taken into account in the projection.

As before, we first examine the ability of the DMD analysis to capture the hydrodynamic motions in the near field. A qualitative assessment of the vortex pairing mechanism is provided by the total spanwise vorticity plots at four equidistant instants during one period in Figure 17. When compared to the field computed directly (Figure 2), we see that both 


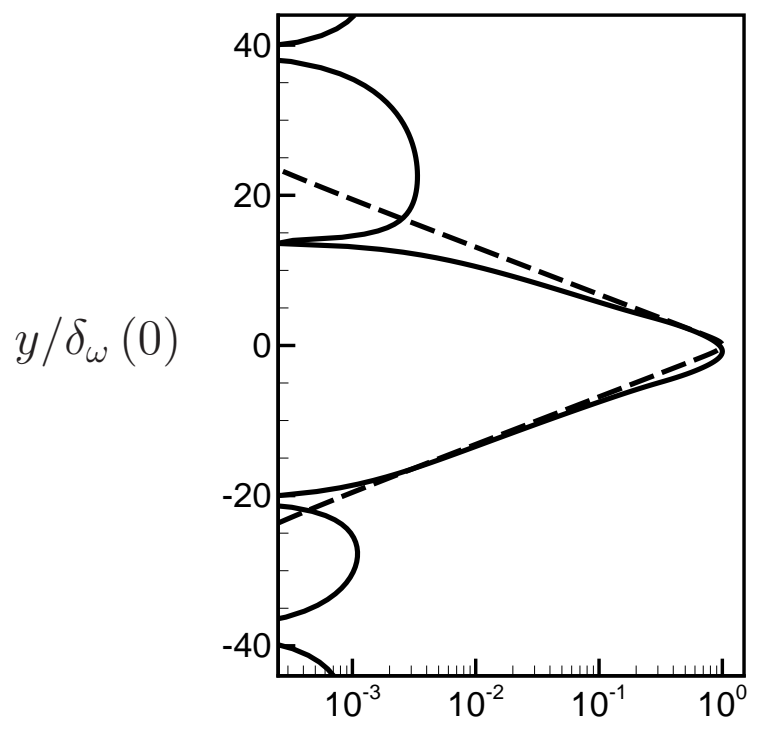

FIG. 16. Comparison of linear and nonlinear models through absolute value of pressure cross sections for the subsonic mixing layer for the subharmonic at $x / \delta_{\omega}(0)=110$ : $(-)$ Koopman mode; (- - - ) global mode. The profiles are made dimensionless by their maximum value.

the roll-up and vortex pairing events are quite well predicted by the DMD analysis. In particular, the projections based on $\mathcal{D}_{s}$ and $\mathcal{D}_{f}$ provide similar results.

In order to further assess the accuracy of the DMD model to represent the near field dynamics, a time signal for the normal velocity field taken at the vortex pairing location $(x=100, y=0) \delta_{\omega_{0}}$ is plotted in Figure 18 by increasing the number of Koopman modes from 1 to 7 (plus their complex conjugate). A very good agreement with NLDE reference is already obtained with only 7 modes for both $\mathcal{D}_{s}$ and $\mathcal{D}_{f}$.

Let us now focus on the acoustic behaviour prediction in the far field. The pressure perturbations from NLDE simulation serving as the reference is plotted in Figure 3(b). The projected fluctuating pressure from DMD is depicted in Figure 19(a). The acoustic patterns are very similar by using the same levels and the acoustic waves emanate from the same apparent source location around $(x, y)=\left(100 \delta_{\omega}(0), 0\right)$. The direction and intensity of acoustic waves can be further validated by plotting the directivity. To this aim, the projected 
Global and Koopman modes analysis of sound generation in mixing layers

(a)

$y / \delta_{\omega}(0)$

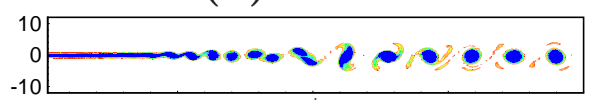

$y / \delta_{\omega}(0)$

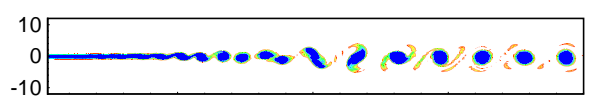

$y / \delta_{\omega}(0)$

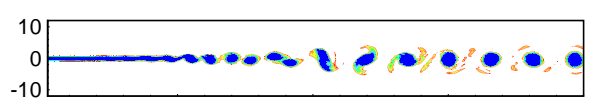

$y / \delta_{\omega}(0)$

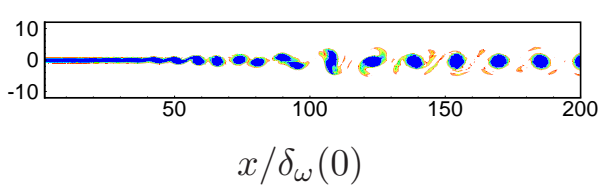

(b)
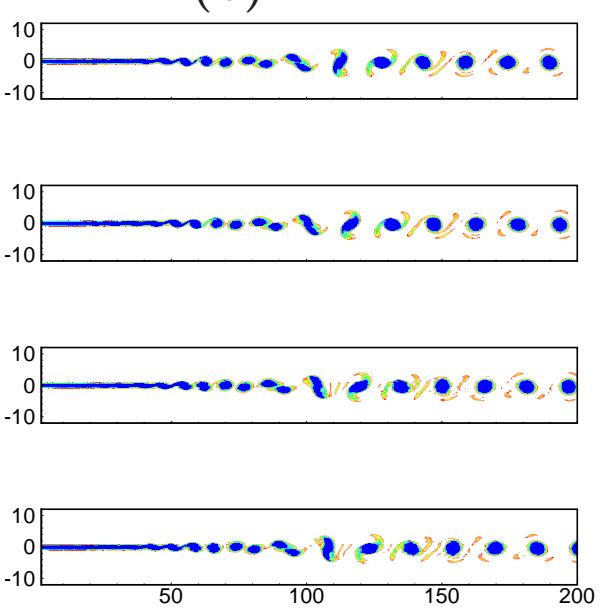

$x / \delta_{\omega}(0)$

FIG. 17. Follow-up of the vortex pairing in the near-field region from a projection onto a Koopmanmode basis with 7 modes associated with: (a) the subdomain region; (b) the entire flow field. Same levels as in Figure 2.

(a)

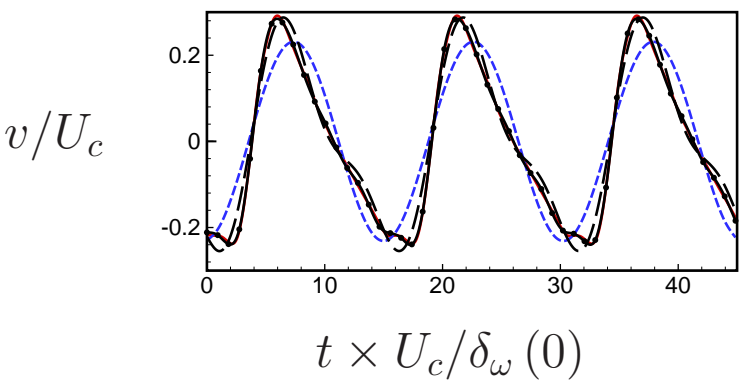

(b)

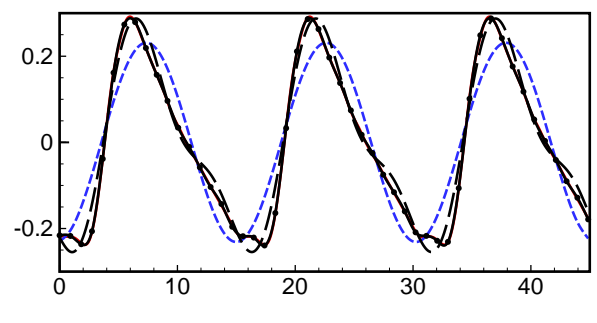

$t \times U_{c} / \delta_{\omega}(0)$

FIG. 18. Temporal evolution of the vertical velocity component taken at $x / \delta_{\omega}(0)=100, y / \delta_{\omega}(0)=$ 0 extracted from a reconstructed flow field onto the dominant Koopman modes over: (a) the entire domain $\mathcal{D}_{s}$; (b) the subdomain $\mathcal{D}_{f}$. Reconstructions with 1 mode $(---) ; 2$ modes $(--)$; 7 modes $(\longrightarrow)$; NLDE results $(-)$.

fluctuating pressure defined by

$$
p^{\prime}(\mathbf{x}, t)=\sum_{k=0}^{m-1} K_{k} p_{k}(\mathbf{x}) e^{-i \omega_{k} t}
$$

with $m-1=13$, is advanced in time during one pairing period to compute the rms values. Figure 19(b) shows a very good agreement with the NLDE both in term of magnitude and 
(a)

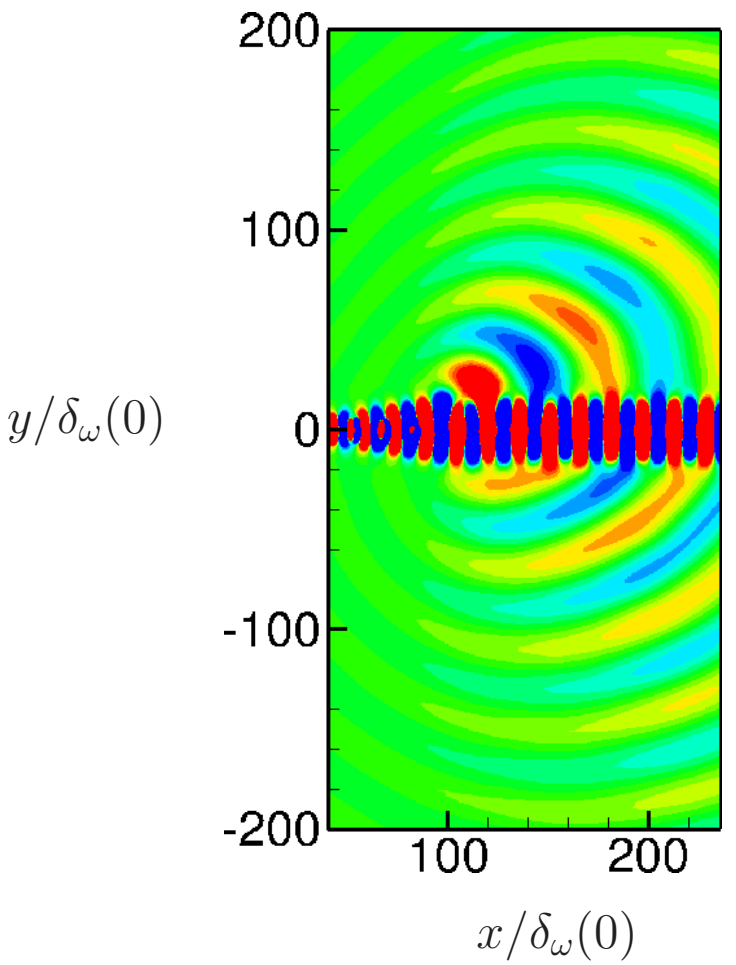

(b)

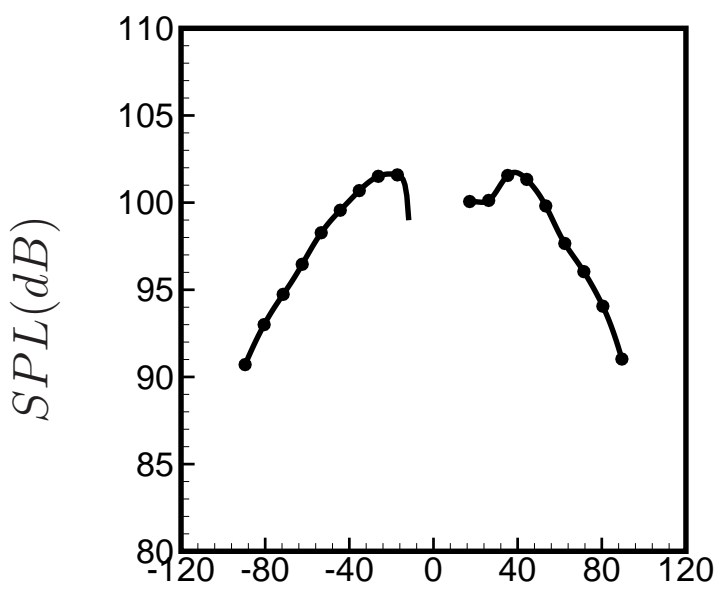

$\theta$

FIG. 19. Reconstruction obtained for 7 Koopman modes: (a) Real part of the pressure fluctuations; (b) Far-field directivity for an arc of radius $100 \delta_{w}(0)$ centered at $x=100 \delta_{\omega}(0)$ : $(-)$ NLDE; $(\rightarrow-)$ Koopman-mode reconstruction.

direction of the wavefronts.

Hence, it is shown that the DMD analysis can provide a low-dimensional representation of the data generated by an aeroacoustic simulation, using a weigthed sum of few eigenfunctions. Moreover, the analysis associated with $\mathcal{D}_{s}$ appears to be sufficiently accurate to be combined with a Kirchhoff extrapolation method to obtain the far-field acoustics. This last remark offers some perspectives in terms of data reduction associated with large aeroacoustic database.

\section{SUPERSONIC CASE}

In this section, the sound generated by Mach wave radiation in a supersonic mixing layer is investigated. 
Global and Koopman modes analysis of sound generation in mixing layers

\section{A. Direct computations}

\section{Problem description}

The case considered is a cold supersonic Mach 2.9/1 mixing layer at a Reynolds number $R e=\rho_{\infty} U_{c} \delta_{\omega}(0) / \mu_{\infty}=1317$, matching one configuration studied in Cheung and Lele ${ }^{8}$. The thermodynamic conditions are the same in the low- and high-speed streams, as described for the subsonic configuration in $\S$ III A 1.

The extent of the computational domain is $\left(L_{x}, L_{y}\right)=(480, \pm 100) \delta_{\omega}(0)$ including the sponge zone. The Cartesian grid has 750 by 331 grid points in the $x$ - and $y$-directions. The grid is uniform in the streamwise direction with spacing $\Delta x_{\min }=0.64 \delta_{\omega}(0)$ up to $x=440 \delta_{\omega}(0)$, and is then stretched with a rate of $4 \%$ to form a sponge zone. Along the normal direction, the minimum mesh size is $\Delta y_{\min }=0.08 \delta_{\omega}(0)$ at the centerline $y=0$, and a stretching with a rate of $2 \%$ is applied up to $y= \pm 100 \delta_{\omega}(0)$.

\section{Base flow}

The procedure to compute the base flow is the same as in the subsonic case. Equations (15) and (16) with a mean convective Mach number $M_{c}=U_{c} / c_{\infty}=1.95$ and a velocity ratio $R=0.487$ are used to prescribe the initial conditions. The full Navier-Stokes equations (1) are advanced without any inlet perturbation. The convergence of residual values for 600000 iterations is depicted in Figure 20(a). A streamwise velocity profile after convergence is compared to the analytical hyperbolic-tangent profile in Figure 20(b). The good correspondance indicates that the perturbations from the local stability analysis in the inlet plane are well supported by the computed base flow.

\section{NLDE computation}

As in the subsonic case, the local linear stability theory allows us to determine the fundamental frequency at the inlet $f_{0}=\omega / 2 \pi$ with $\omega$ the circular frequency $\approx 2 \pi \times 0.286 U_{c} / \delta_{\omega}(0)$ (see Appendix A). To illustrate the sound generated by the instability waves in a supersonic regime, the flow is forced at the fundamental frequency at each time step.

The simulation is run for 50000 iterations with $\delta t U_{c} / \delta_{\omega}(0) \simeq 0.043$. After the transient 
(a)

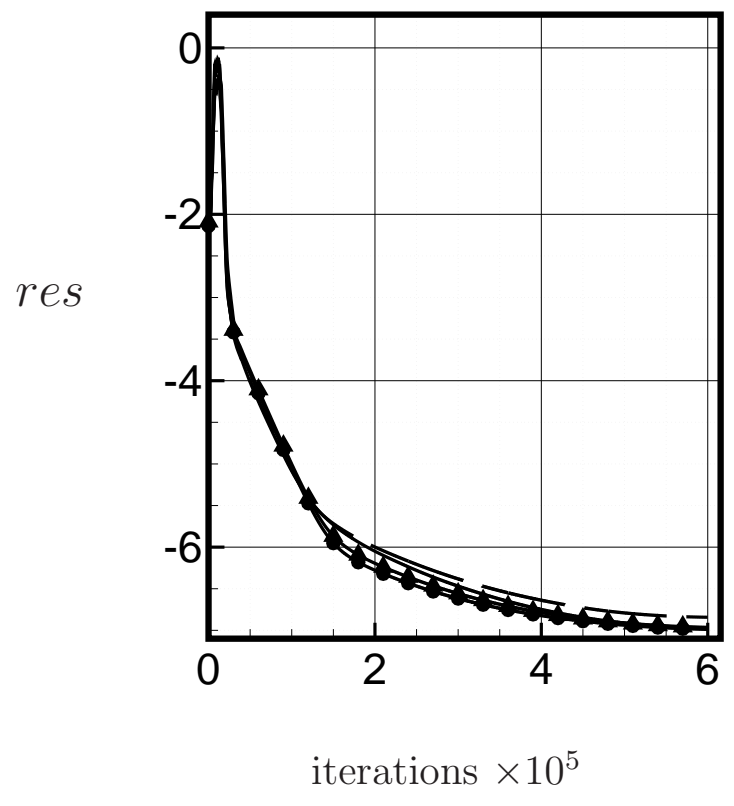

(b)

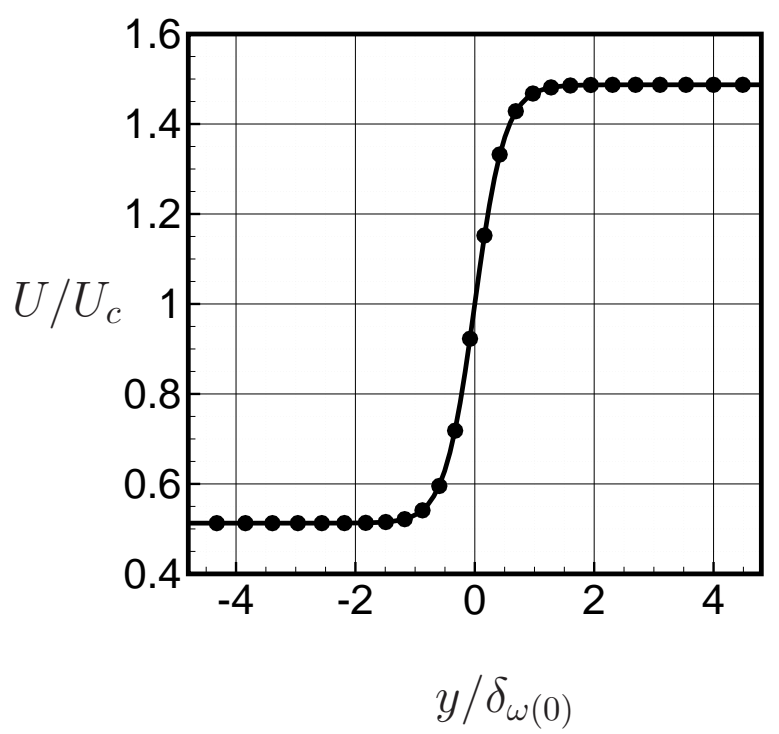

FIG. 20. Base flow for the supersonic mixing layer: (a) convergence of normalized residuals for the conservative variables as a function of time iterations (see figure 1 for line legend); (b) comparison of streamwise velocity profiles of the base flow $(\bullet \bullet)$ and the hyperbolic-tangent function $(15)(-$ ) at the inlet plane.

regime, the near field vorticity is displayed in Figure 21(b) which lead to pressure fluctuations as depicted in Figure 21(a).

In the absence of vortex pairing, the radiation pattern illustrates Mach wave radiation in the lower stream as observed by Cheung \& Lele ${ }^{8}$ in a similar supersonic mixing layer. This behaviour is in agreement with the fact that the fundamental frequency has a subsonic relative phase speed in the upper stream and a supersonic phase speed in the lower stream (see Cheung \& Lele ${ }^{8}$ ). To examine, how the linear model based on a global modes decomposition can capture the instability waves and the acoustic behaviour observed in the NLDE simulation, a global linear stability analysis is performed in the next section.

The evolution of the integrated energy at the frequencies $f_{0}$ and $2 f_{0}$ is shown in Figure 22. The fundamental mode forced at the inlet first undergoes an exponential growth and then grows with a slower slope due to nonlinear saturation. The first harmonic is not forced and has a very low initial amplitude. It then grows at a greater rate due to nonlinear interactions. 
(a)

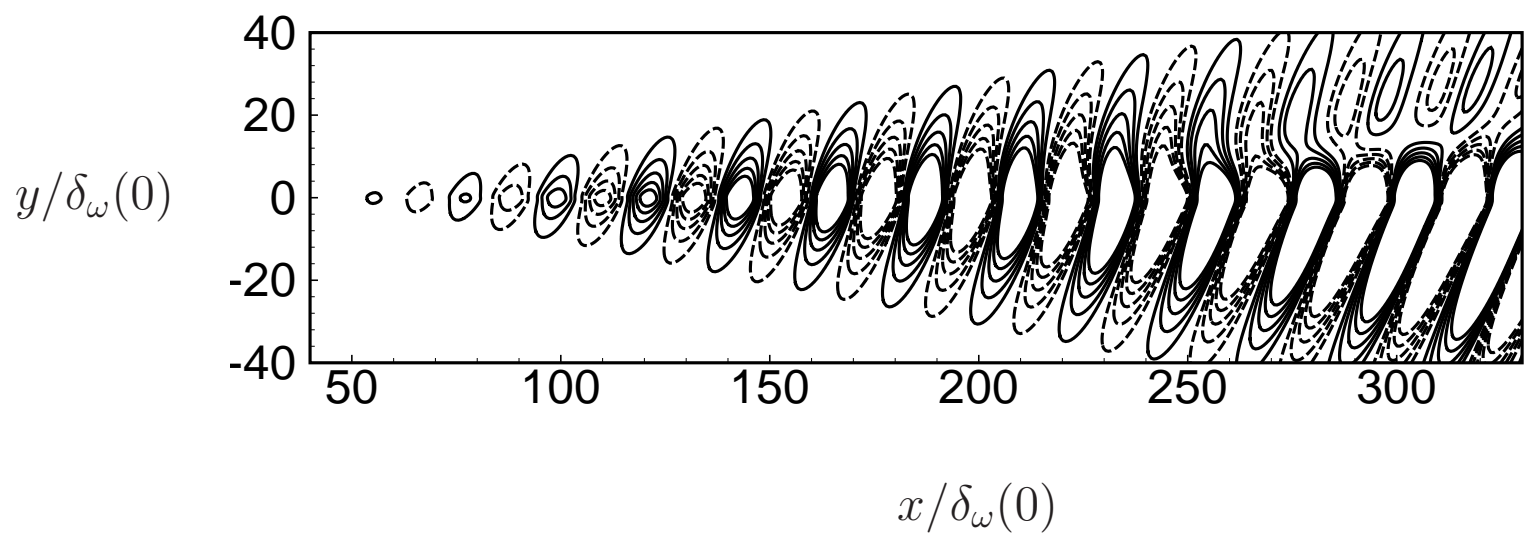

(b)

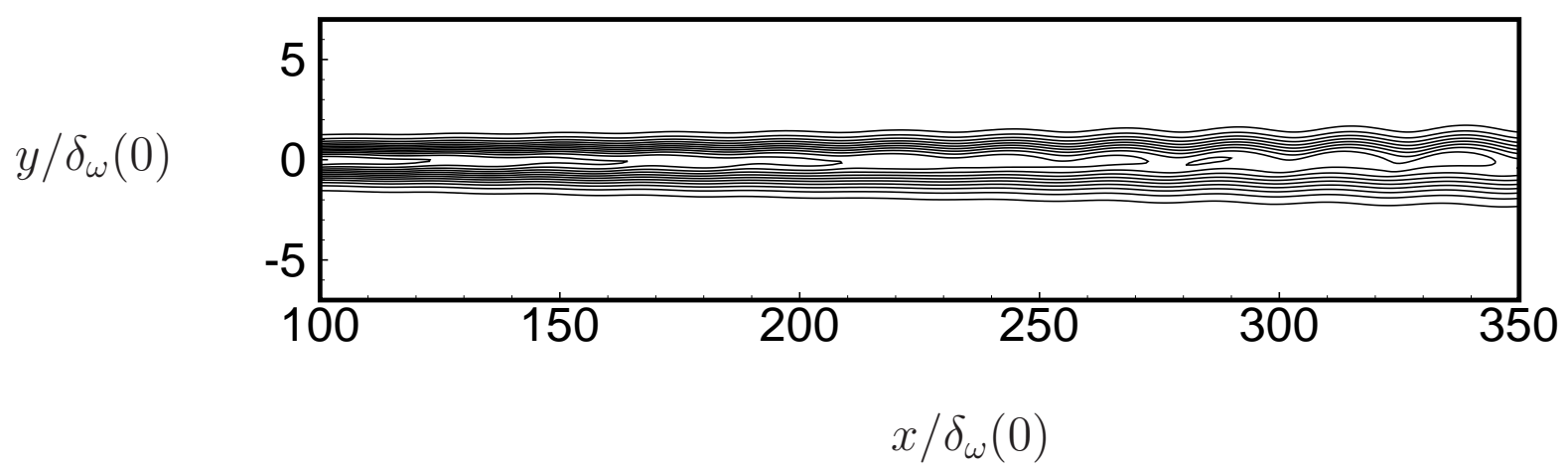

FIG. 21. (a) Far-field non-dimensional fluctuating pressure field with contours levels from -0.01 to 0.01 in steps of 0.002 (full lines: positive values, dashed lines: negatives values). (b) Total non-dimensional spanwise vorticity with contours ranging from 0.05 to 1.5 in steps of 0.05 .

\section{B. Linear model: global spectrum, eigenfunctions}

The initial perturbation described in the subsonic case is also used for the supersonic regime. It is initially located at $\left(x_{0}, y_{0}\right)=\left(40 \delta_{\omega}(0), 0\right)$. The snapshots are carried out for a window extending from $x=10 \delta_{\omega}(0)$ to $x=340 \delta_{\omega}(0)$ and $y= \pm 75 \delta_{\omega}(0)$. The global modes are obtained with a sequence of 380 snapshots which are obtained with a sampling frequency which satisfies a resolution up to $2 f_{0}$ in agreement with the Nyquist criterion.

The dimensionless eigenvalues are presented in Figure 23. One may observe that all the global modes are damped temporally, which is consistent with the globally stable property of such a flow. In Figure 24, the real part of the pressure fluctuation is shown for $\omega_{r} \approx 0.3$, which is closed to the forcing frequency used in the nonlinear regime $\left(\omega_{r} \approx 0.28\right)$, and for 
Global and Koopman modes analysis of sound generation in mixing layers

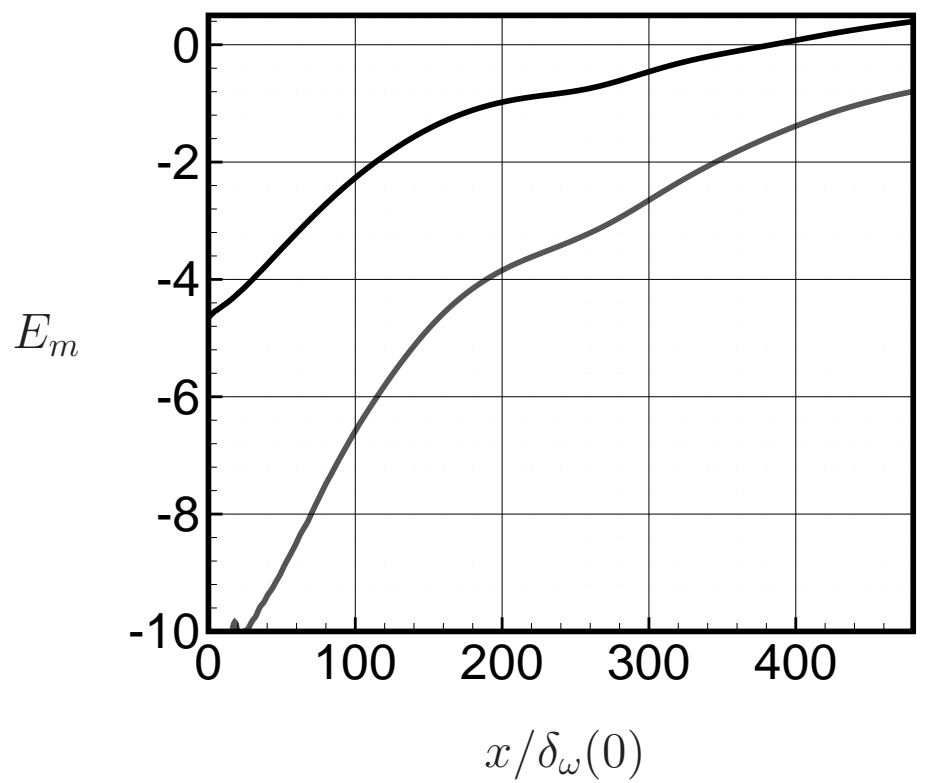

FIG. 22. NLDE computation for the supersonic case. Integrated modal energy for $f_{0}(-)$ and $2 f_{0}(-)$.

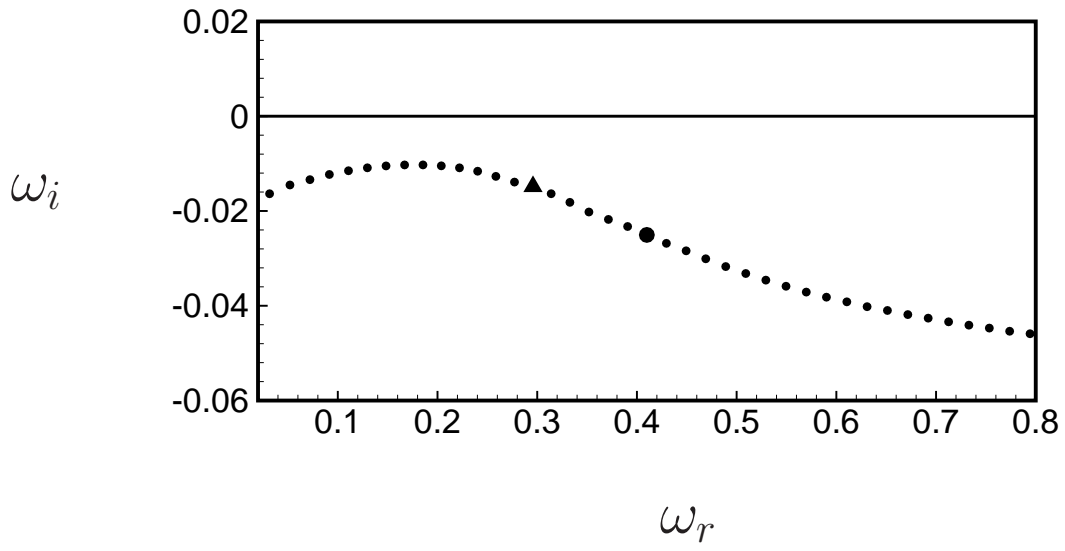

FIG. 23. Global spectrum of the supersonic mixing layer. The eigenvalues are made dimensionless by $\delta_{\omega}(0)$ and $U_{c}$.

$\omega_{r} \approx 0.4$

It is worth noting that a strong Mach wave radiation in the lower stream is also evidenced for these global modes. To illustrate the latter point, the phase velocity relative to the ambient sound speed for both circular frequencies is determine under the weakly nonparallel flow assumption. In Figure 25, these quantites are plotted for the vertical velocity fluctuation taken at $y=0$. Despite some ocillations, the phase velocity is clearly supersonic for the 
(a)

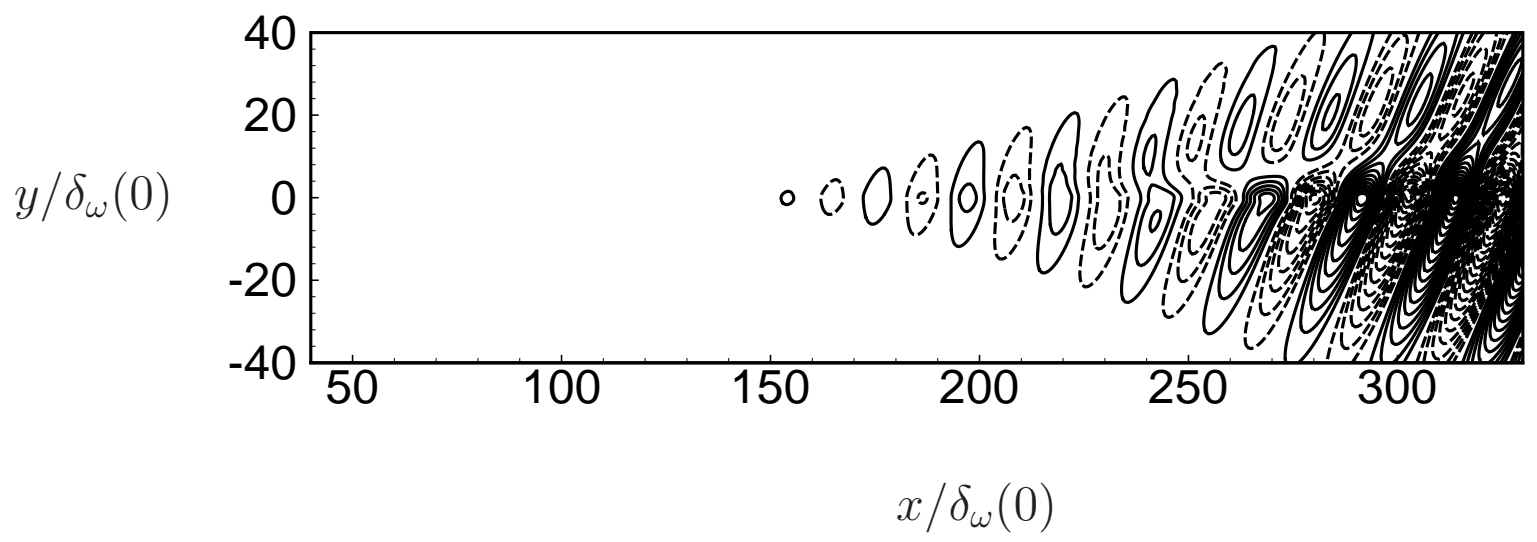

(b)

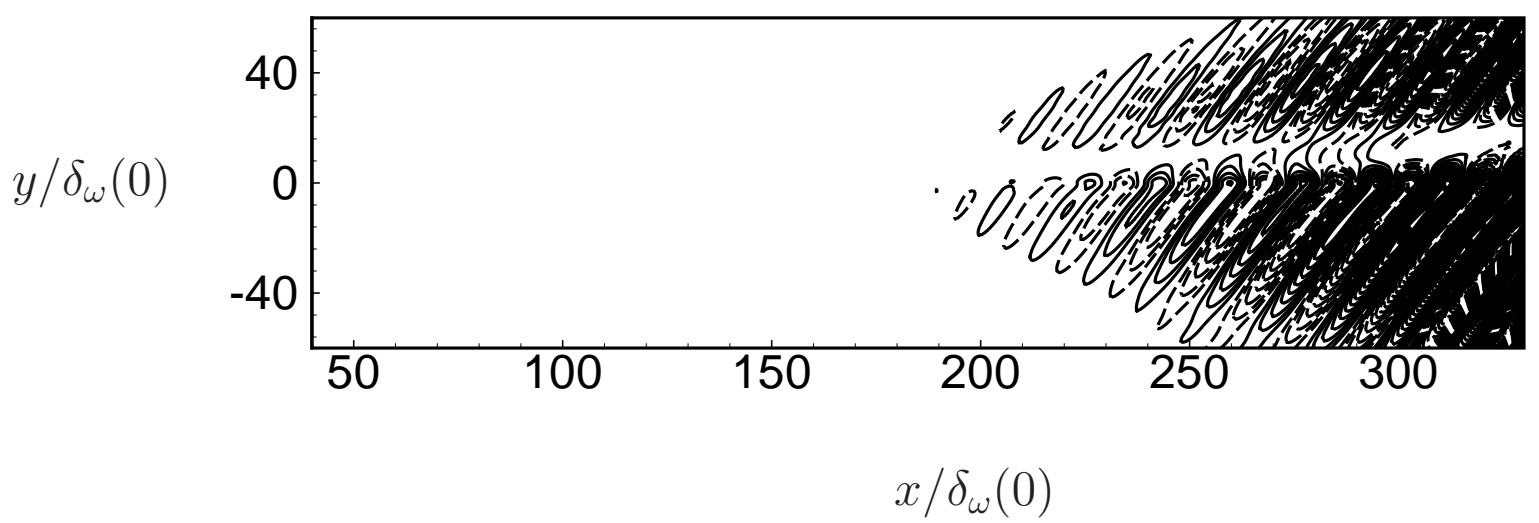

FIG. 24. Real part of the pressure component for two global eigenmodes corresponding to (a) $\boldsymbol{\Delta}$ and (b) • in the spectrum of Figure 23. Full lines: positive values; dashed lines: negatives values.

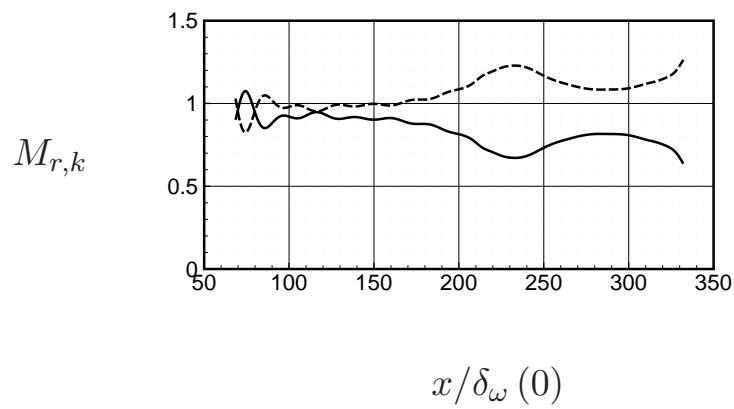

(a)

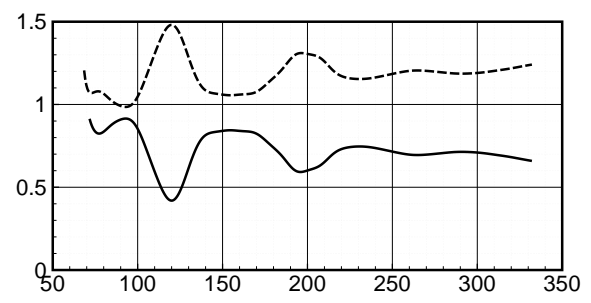

$x / \delta_{\omega}(0)$

FIG. 25. Phase speeds $M_{r, 1}(-)$ and $M_{r, 2}(-\quad-\quad$ ) of the two global eigenmodes marked by: (a) $\boldsymbol{\Delta}$ and (b) • in the spectrum of Figure 23. 
Global and Koopman modes analysis of sound generation in mixing layers

lower stream, while the fluctuations in the upper stream are moving with a subsonic phase speed for both frequencies. Consequently, these linear instability waves will lead to acoustic radiation. Finally, let us further explore the angle of the Mach wave radiation measured from the upstream axis for the lower stream. For the global mode whose frequency is closed to the forcing frequency, the Mach angle is estimated at $53^{\circ}$ near $x / \delta_{\omega}(0) \approx 325$, in good agreement with the NLDE value. Strong similarities can be noticed between the global mode in Figure 24(a) and the NLDE simulation in Figure 21(a).

Hence, the solution derived from a linear global stability analysis with appropriate nonreflecting boundary conditions gives a series of discrete supersonic waves, which radiates sound directly through Mach wave mechanism. This supports the weakly nonparallel theory based on matched asymptotic expansions of Tam \& Burton ${ }^{47}$ dedicated to the noise generated by spatially growing instability in supersonic mixing layers.

Finally, as discussed in a subsonic regime, these discrete instability waves may be used to represent the effect of a localized harmonic forcing through an appropriate summation (22). Since each individual global mode may radiate sound, the asymptotic response derived from nonmodal interaction will produce some noise.

\section{Nonlinear model: Koopman modes}

A DMD analysis is performed on the NLDE database described in section IV A 3 by taking the entire domain. A sequence of 210 snapshots is recorded with a sampling period which satisfies the Nyquist criterion up to $6 f_{0}$. The energy spectrum for dominant modes and the corresponding Ritz values are displayed in Figure 26.

Due to the saturated regime characterizing the NLDE database, the Ritz values are seen to lie on a unit circle in Figure 26(b). The most observable modes are associated with the energy peaks shown in Figure 26(a). As expected, the flow dynamics is mainly dominated by the fondamental forcing frequency and its first harmonics. In addition, the energy spectrum shown in Figure 26(a) clearly exhibits a different magnitude distribution for the dominant Koopman modes compared to the one obtained for the subsonic regime. In particular, the influence of the nonlinear effects are weaker which results in a small contribution of the harmonics into the dynamics in comparison with the fundamental frequency. Furthermore, the zero frequency mode contribution is almost undistinguishable. This provides some insight 
(a)

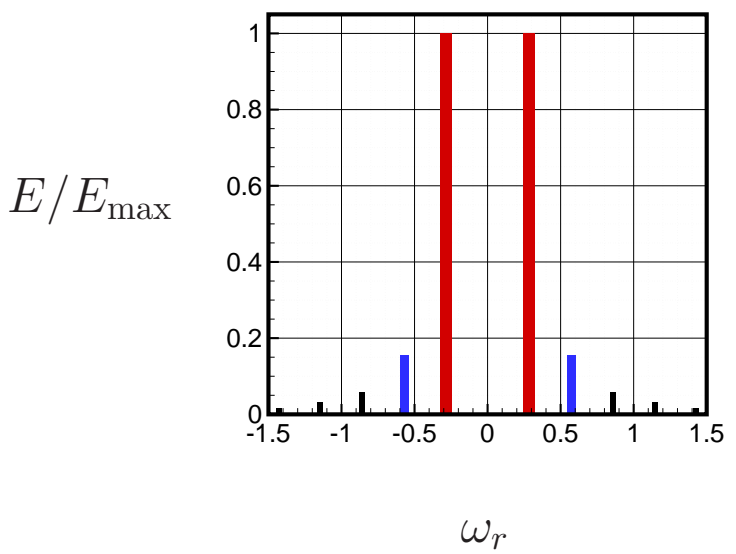

(b)

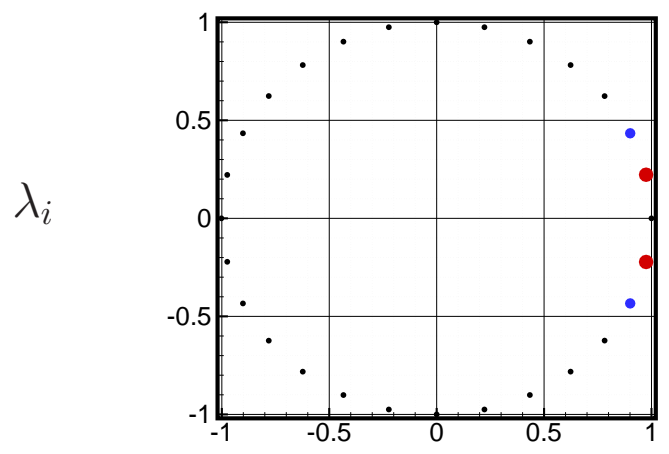

$\lambda_{r}$

FIG. 26. DMD analysis for the supersonic mixing layer: (a) Energy spectrum; (b) Ritz values. The values are made dimensionless by $\delta_{\omega}(0)$ and $U_{c}$.

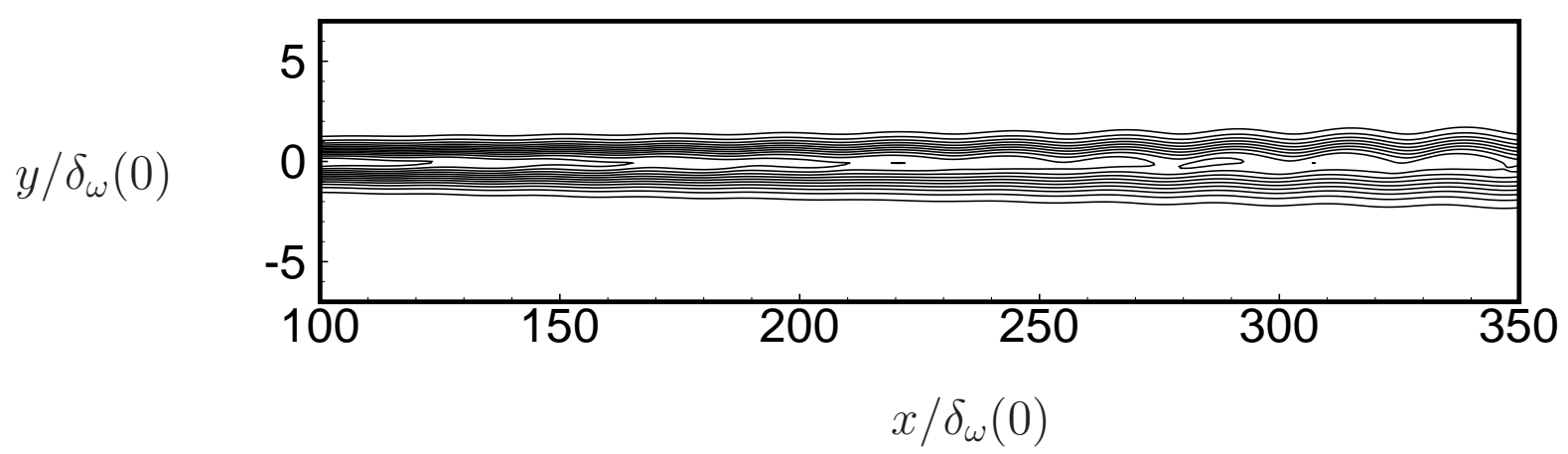

FIG. 27. Total non-dimensional spanwise vorticity based on the projection of an instantaneous snapshot from NLDE onto the Koopman modes which correspond to $f_{0}$ and $2 f_{0}$. Contours range from 0.05 to 1.5 in steps of 0.05 .

into the results depicted in Figure 22 where nonlinear effects will lead to a saturation of the instability waves with almost no mean flow modification.

The ability of the Koopman modes to capture the near field structures is highlighted through a reconstruction of the entire flow field based on a projection of the flow dynamics onto the two dominant Koopman modes, $f_{0}$ and $2 f_{0}$ and their complex conjugates. The total vorticity plotted in Figure 27 is almost identical to the NLDE result of Figure 21(b). This clearly demonstrates the ability of the Koopman modes to reproduce the near field 
(a)

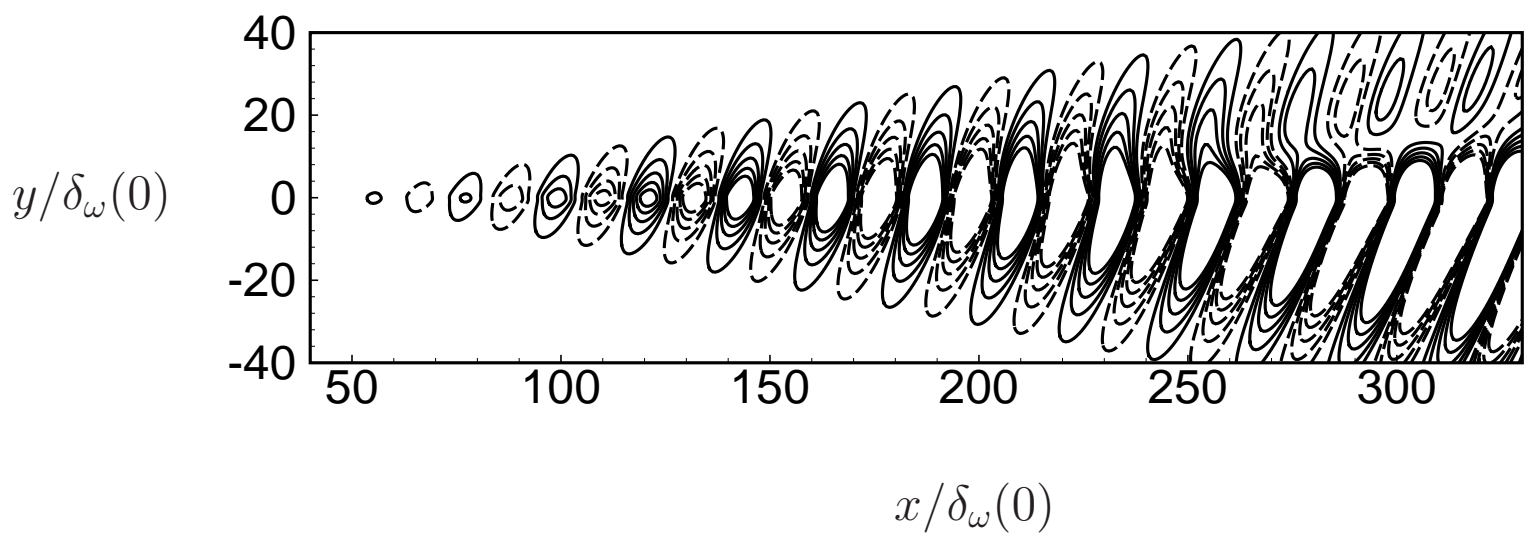

(b)

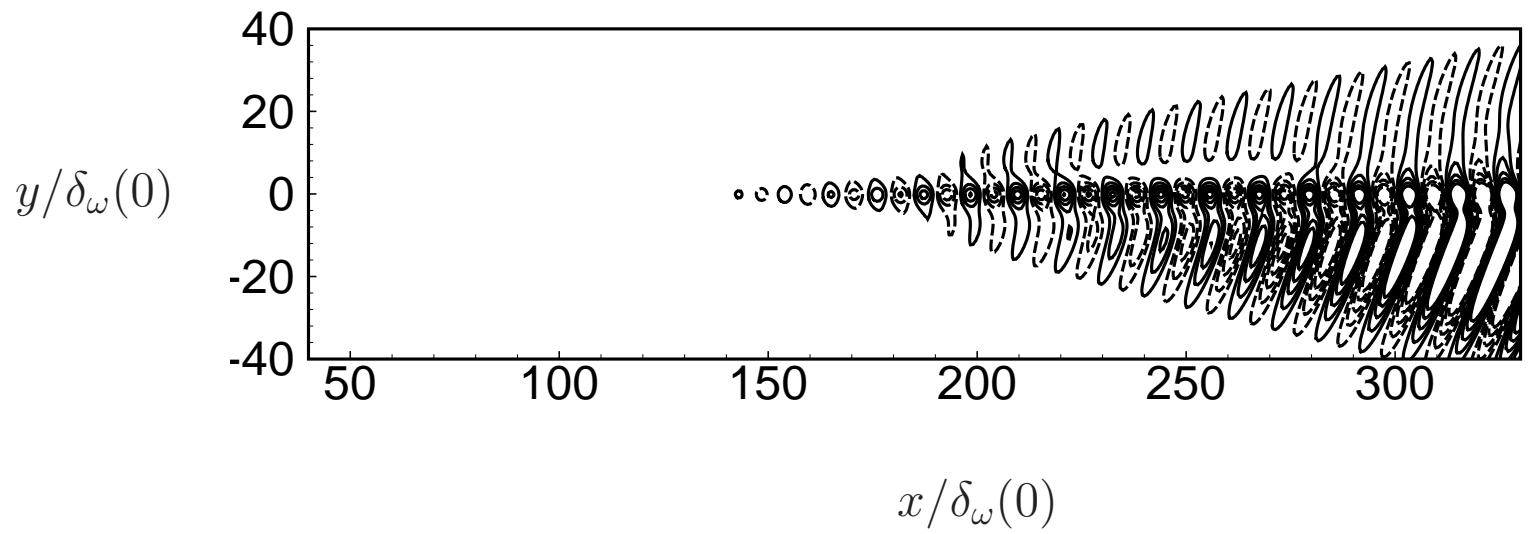

FIG. 28. Non-dimensional pressure component of Koopman modes projected onto an instantaneous snapshot from NLDE: (a) $f_{0}$ levels range from -0.01 to 0.01 ; (b) $2 f_{0}$ levels range from -0.002 to 0.002 .

dynamics.

Concerning the acoustic behaviour, the pressure fields for the fundamental and its first harmonic are displayed in Figure 28. It appears that both the fundamental and its harmonics generate Mach wave radiation on the lower stream. These waves also propagate in the upper side but with a lower amplitude and interferences arise due to the propagation effects through the shear zone. In particular, the strong similarity between the Koopman mode for $f_{0}$ and the NLDE simulation is conform with the hierarchy in the energy spectrum of Figure 26(a). In addition, the radiated noise for the Koopman mode $f_{0}$ closely follows the results obtained with the linear global stability analysis. In particular, the Mach angle in the lower stream and the interference pattern in the upper stream are in agreement with the global mode 


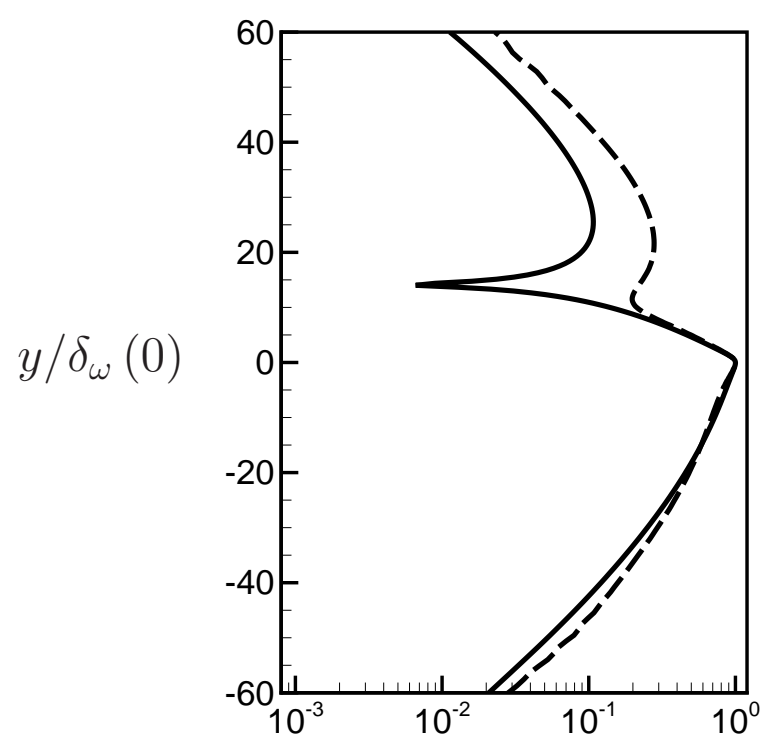

FIG. 29. Comparison of linear and nonlinear models through absolute value of pressure cross sections for the supersonic mixing layer for the fundamental frequency at $x / \delta_{\omega}(0)=300$ :

Koopman mode; (- - - ) global mode. The profiles are made dimensionless by their maximum value.

analysis.

To further illustrate the last comment, cross-sections along the normal direction for the modulus of the fluctuating pressure are extracted at $x / \delta_{\omega}(0)=300$ for the fundamental Koopman mode as well as the global mode which corresponds to $\omega_{r} \approx 0.3$ (close to $f_{0}$ ). In Figure 29, in contrast to the subsonic case, we can see that the strength of the radiated pressure in lower stream for the global mode is consistent with the Koopman mode. In addition, the pattern radiated in the upper stream exhibits also similarities. The difference in amplitude visible in the upper side indicates discrepancies when the Mach waves propagate through the shear region. The linear mode can indeed not represent the saturated vortices. Overall, these results clearly show that in a supersonic regime, the radiated pressure is directly linked to the linear spatially growing instability wave which can be represented with a linear global-mode analysis. 
Global and Koopman modes analysis of sound generation in mixing layers

\section{CONCLUSIONS AND OUTLOOK}

In this paper, the linear and nonlinear mechanisms for noise generation are revisited from a global point of view. In this context, Krylov's methods based on snapshots sampled from linear and nonlinear simulations are employed to extract the dominant global modes and Koopman modes, respectively. Forced two-dimensional mixing layers are investigated for two different flow regimes. The subsonic regime is characterized by pairing events at a fixed location, whereas Mach wave radiation is possible in the supersonic regime. First, a base flow is obtained from the solution of the full Navier-Stokes solver and is used in both linear and nonlinear disturbance equation (LDE and NLDE) solvers. This formalism allows the use of exactly the same discretization in all methods carried out for the flow analysis.

In the subsonic regime, a linear global analysis allows a fair characterization of the KelvinHelmholtz instabilities, considering the low Reynolds number of the flow. In a global framework, these convective modes are stable and the wavepacket dynamics can be retrieved through the response to an external forcing. The individual modes are not radiating sound and the noise generation mechanism can not be reproduced since it relies on the deformation and acceleration of saturated vortices during the pairing event. By contrast, a dynamic mode decomposition (DMD) based on the NLDE database yields an approximation of the Koopman mode which contains the full dynamical information. It is shown in particular that the mixing layer flow and the associated noise can be reproduced with few DMD modes. The accuracy of the modal decomposition is demonstrated by using snapshots defined on a subdomain only restricted to the shear region. The far field radiation can be faithfully evaluated with a Kirchhhoff's wave extrapolation method, indicating that all the compressibility effects are represented by the subdomain modes.

In the supersonic regime, the phase speed of disturbances relative to the ambient sound speed can be supersonic and linear instabilities directly radiate sound, the so-called Mach waves, as shown by previous weakly non-parallel stability theories. Consequently, the eigenfunctions obtained from a linear global analysis highlight Mach wave radiation which shows good agreement with a direct computation. However, the linear analysis fails to reproduce the complex interferences in the shear zone, associated with subtle details of the instantaneous nonlinear flow. On the other hand, the Koopman mode analysis allows a correct calculation of both near and far fields. The interference pattern visible in the upper stream 
Global and Koopman modes analysis of sound generation in mixing layers

is notably well reproduced.

Hence, the present analysis provides a general strategy to determine the linear and nonlinear noise generation mechanism associated with instability waves where classical methods, such as PSE or multiple scales techniques, are inapropriate and/or inapplicable. For instance, it should be particularly interesting to apply such a strategy in heated or counterflow mixing layers ${ }^{46}$, subsonic and supersonic hot jets ${ }^{44}$ or impinging jets ${ }^{23}$ where upstream propagating waves may occur and more generally for three-dimensional and turbulent flows. For the latter, efficient algorithms are needed for the extraction of the dominant modes to deal with the storage of data collected by sampling the numerical simulation. On one hand, one may suggest to improve the efficiency of the DMD algorithm by exploiting the parallel structure of the numerical simulation code. On the other hand, one may also process only subdomains of the entire flow field. In the framework of aeroacoustics, the revelancy of such a strategy is highlighted in the present work. For turbulent flows, a similar analysis which focuses on low frequency unsteadiness in shockwave turbulent boundary layer interaction is carried out by Grilli et al. ${ }^{22}$. By processing only subdomains of the numerical simulation, Grilli et al. ${ }^{22}$ show that the essential characterisitics of the low-frequency dynamics may be reduced to the superposition of the four-dominant low-frequency Koopman modes. Finally, the recent DMD analysis performed on the H-type transition to turbulence in a flat-plate boundary layer by Sayadi et al. ${ }^{40}$ allows the identification of three-dimensional large-scale coherent structures which are responsible for the main contribution to the wall skin-friction. For that purpose, a composite DMD which rearranges quantities of different units into the column vectors associated with the data sequence of snapshots is used by the authors. Hence, a composite DMD combining sound pressure fluctuation measurement in the far-field and large-scale coherent structures in the near-field could be an interesting prospect of the present analysis aiming at identifying the contribution of vortical structures onto the radiated noise for complex turbulent flows.

\section{Appendix A: Local linear stability analysis of the inlet plane}

The disturbance vector is

$$
\mathbf{q}^{\prime}(x, y, t)=\hat{\mathbf{q}}(y) e^{i(\alpha x-\omega t)}+c . c .
$$


Global and Koopman modes analysis of sound generation in mixing layers

(a)

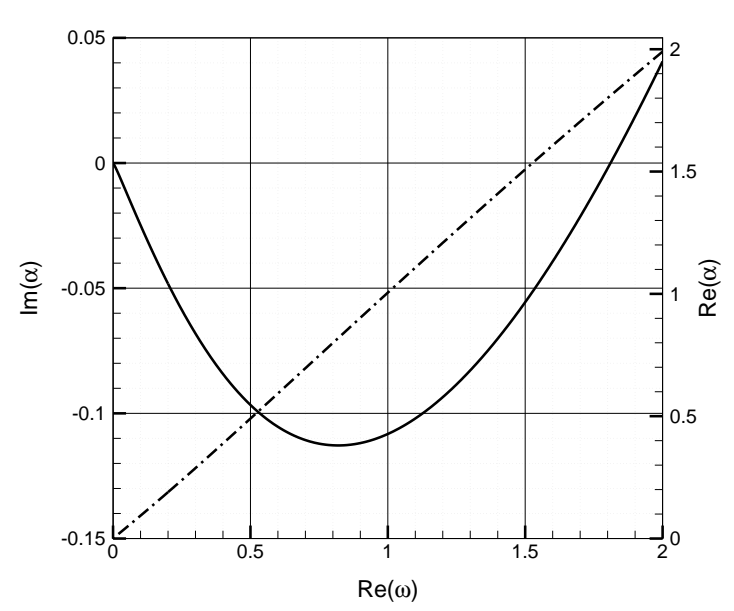

(b)

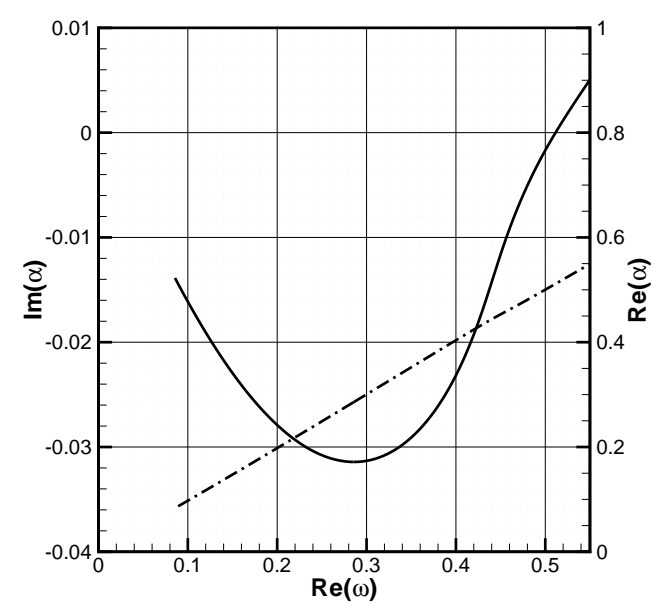

FIG. 30. Dispersion relation: (a) subsonic and (b) supersonic mixing layers.

with $\alpha=\alpha_{r}+i \alpha_{i}$ the complex wavenumber and $\omega$ the real circular frequency. Only twodimensional modes are considered even if oblique modes can be more unstable at supersonic speeds since the test cases are $2 \mathrm{D}$. The local spatial stability theory can be rewritten into a generalized eigenvalue problem:

$$
\left[\mathbf{C}_{0}+\mathbf{C}_{1} \alpha+\mathbf{C}_{2} \alpha^{2}\right] \hat{\mathbf{q}}=0, \text { with } \hat{\mathbf{q}}=(\hat{\rho}, \hat{u}, \hat{v}, \hat{p})
$$

The eigenproblem is solved using a Chebyshev collocation spectral method and a QZ algorithm. The base flow is approximated by the streamwise velocity profile (15), which correctly approximates the solution for self-similar boundary-layer equations. The Crocco-Buseman relationship (16) is used for the temperature profile.

Both real and imaginary parts of $\alpha$ are plotted as a function of $\omega$ in Figure 30. The spatial amplification rate is given by $-\alpha_{i}$. Hence, the most unstable mode is obtained for the circular frequency which leads to the largest spatial amplification rate. The inlet flow parameters are summarized in Table I. The shape of the corresponding eigenfunctions are shown in Figure 31. Since the analysis is linear, we need to prescribe an amplitude for the inlet perturbations, as $\mathbf{q}_{\text {in }}^{\prime}=\varepsilon \mathbf{q}^{\prime}$ with $\mathbf{q}^{\prime}$ given by (A1) and values of $\varepsilon$ are reported in Table I. 
Global and Koopman modes analysis of sound generation in mixing layers

\begin{tabular}{|c|c|c|c|c|c|c|c|c|c|c|}
\hline Case & $M_{1}$ & $M_{2}$ & $M_{c}$ & $R e$ & $\delta_{\omega}(0) / \mathrm{m}$ & Mode & $\alpha_{r}$ & $\alpha_{i}$ & $\omega$ & $\varepsilon$ \\
\hline \hline Subsonic & 0.5 & 0.25 & 0.375 & 1500 & $1.6710^{-4}$ & $\begin{array}{l}\text { fundamental } \\
\text { sub-harmonic }\end{array}$ & 0.8234 & -0.1117 & 0.8235 & -0.0849 \\
0.4117 & $10^{-4}$ \\
\hline Supersonic & 2.9 & 1. & 1.95 & 1317 & $2.8210^{-5}$ & fundamental & 0.2860 & -0.0314 & 0.2860 & $410^{-5}$ \\
\hline
\end{tabular}

TABLE I. Numerical parameters used for the boundary condition at the inlet for the subsonic and the supersonic mixing layer. $\varepsilon$ denotes the forcing amplitude.

(a)

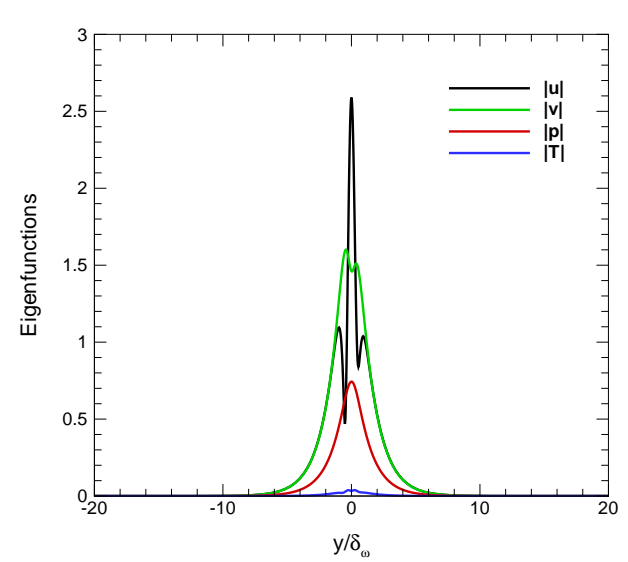

(b)

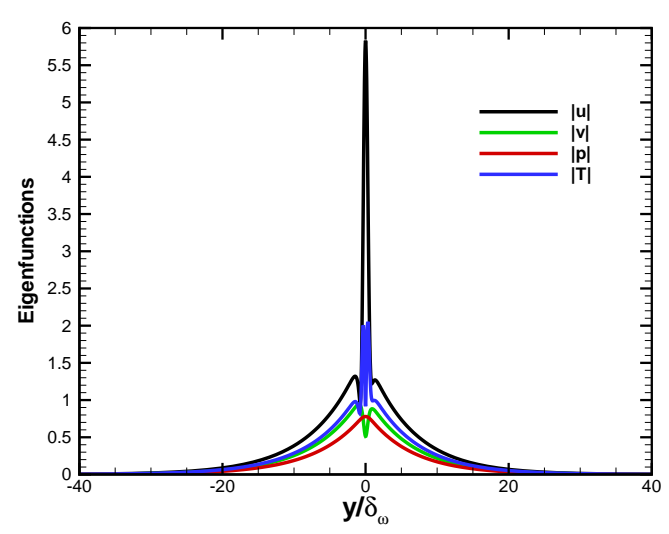

FIG. 31. Eigenfunctions for the most amplified wave: (a) subsonic and (b) supersonic mixing layers.

\section{Appendix B: Convected Kirchhoff wave extrapolation method}

To evaluate far-field noise from the near-field region, a wave extrapolation method based on Kirchhoff's formulation ${ }^{26}$ is used. One may consider a surface $\Sigma$, defined by the equation $f(\mathbf{x}, \mathbf{t})=0$, which encloses the source region. The effect of the mean flow in the observation region is taken into account by considering the convected wave operator for uniform streams with Mach numbers $M$ set at the values of the lower or upper streams for the mixing layer case. The 2-D frequency-domain convected form of the Kirchhoff formulation can be written 
Global and Koopman modes analysis of sound generation in mixing layers

as

$$
\begin{gathered}
H(f) p^{\prime}(\mathbf{x}, \omega)=\frac{i \beta}{4} \int_{f=0}\left\{\frac{\partial p(\mathbf{y}, \omega)}{\partial \mathbf{n}_{\beta}} \mathrm{H}_{0}^{(2)}\left(\frac{k}{\beta^{2}} r_{\beta}\right)+\frac{k}{\beta^{2}} p(\mathbf{y}, \omega)\left[\frac{\partial r_{\beta}}{\partial \mathbf{n}_{\beta}} \mathrm{H}_{1}^{(2)}\left(\frac{k}{\beta^{2}} r_{\beta}\right)-\right.\right. \\
\left.\left.i M \frac{\partial y_{1}}{\partial \mathbf{n}_{\beta}} \mathrm{H}_{0}^{(2)}\right]\right\} \exp \left(i \frac{M k\left(x_{1}-y_{1}\right)}{\beta^{2}}\right) \mathrm{d} \Sigma_{\beta}
\end{gathered}
$$

where $\omega$ and $k=\omega / c_{\infty}$ are the circular frequency and the wavenumber. $\mathbf{x}$ denotes the observer position and $\mathbf{y}$ is a source point on the extrapolation surface $\Sigma$. $\mathrm{H}_{0}^{(2)}$ and $\mathrm{H}_{1}^{(2)}$ are the Hankel functions of second kind of zeroth and first order, respectively. The Prandtl-Glauert transformation is employed with $\beta=\sqrt{1-M^{2}}$ and $r_{\beta}=\sqrt{\left(x_{1}-y_{1}\right)^{2}+\beta^{2}\left(x_{2}-y_{2}\right)^{2}}$. The notation $\partial / \partial \mathbf{n}_{\beta}$ means $\left(\partial / \partial y_{i}\right) n_{i \beta}$, where $n_{1 \beta}=n_{1}, n_{2 \beta}=\beta n_{2}$ and $n_{i}$ are the component of the unit outer normal $\mathbf{n}$. Similarly, $\mathrm{d} \Sigma_{\beta}$ is used for the Prandtl-Glauert transform of the surface element. $H(f)$ denotes the Heaviside function, zero inside the extrapolation surface $(f<0)$ and one outside $(f>0)$. Details of this frequency-domain formulation are provided in $^{19}$.

From an algorithmic point of view, the first step is the storage of the aerodynamic quantities during one vortex pairing of the simulation or the reduced order model. In particular, the variable $p$ is recorded onto a horizontal surface surrounding the source region. Then, the normal derivatives $\partial p / \partial y_{2}$ is evaluated from the near-field solution with the same DRP scheme used for the direct solver in section II A. Fast Fourier transforms of $p$ and $\partial p / \partial y_{2}$ allow us to obtain the sound generated in the far field by integrating (B1) with a trapezoidal rule. Finally, the pressure $p^{\prime}(\mathbf{x}, t)$ in the temporal space is retrieved by an inverse Fourier transform. 
Global and Koopman modes analysis of sound generation in mixing layers

\section{REFERENCES}

${ }^{1}$ F. Alizard and J.-C. Robinet. Modeling of optimal perturbations in flat plate boundary layer using global modes: benefits and limits. Theor. Comp. Fluid Dyn., 1-4(25):147-165, 2011.

${ }^{2}$ F. Alizard, J.-C. Robinet, and X. Gloerfelt. A domain decomposition matrix-free method for global linear stability. Computers and Fluids, 1-4(25):147-165, 2012.

${ }^{3}$ S. Bagheri, E. Akervik, L. Brandt, and D. S. Henningson. Matrix-free methods for the stability and control of boundary layers. AIAA J., 47(5):1057-1068, 2009.

${ }^{4}$ W. Blumen. Shear-layer instability of an inviscid compressible fluid. J. Fluid Mech., 40:769-781, 1970.

${ }^{5}$ W. Blumen, P. G. Drazin, and D. F. Billings. Shear-layer instability of an inviscid compressible fluid: Part 2. J. Fluid Mech., 71:305-316, 1975.

${ }^{6}$ C. Bogey and C. Bailly. A family of low dispersive and low dissipative explicit schemes for noise computation. J. Comp. Physics, 194:194-214, 2004.

${ }^{7}$ C. Bogey, C. Bailly, and D. Juvé. Numerical simulation of sound generated by vortex pairing in a mixing layer. AIAA Journal, 38(12):2210-2218, 2000.

${ }^{8}$ L. C. Cheung and S. K. Lele. Linear and nonlinear processes in two-dimensional mixing layer dynamics and sound generation. J. Fluid Mech., 625:321-351, 2009.

${ }^{9}$ T. Chyczewski, P. Morris, and L. Long. Large-eddy simulation of wall-bounded shear flow using the nonlinear disturbance equations. In 6th AIAA/CEAS Aeroacoustics Conference, 12-14 June, Lahaina, Hawaii, AIAA Paper 2000-2007, 2008.

${ }^{10}$ T. Colonius, SK. Lele, and P. Moin. Sound generation in a mixing layer. J. Fluid Mech., 330:2761-2778, 1997.

${ }^{11}$ C. Cossu and J-M. Chomaz. Global measures of local convective instabilities. Phys. Rev. Lett., 78(23):4387-4390, 1997.

${ }^{12} \mathrm{Ph}$. Meliga D. Sipp, O. Marquet and A. Barbagallo. Dynamics and control of global instabilities in open flows: a linearized approach. Appl. Mech. Rev., 63:030801, 2010.

${ }^{13}$ M. Fosas de Pando, D. Sipp, and P.J. Schmid. Efficient evaluation of the direct and adjoint linearized dynamics from compressible flow solvers. J. Comp. Physics, 231:7739$7755,2012$.

${ }^{14}$ P. Druault, M. Yu, and P. Sagaut. Quadratic stochatic estimation of far field acoustic 
Global and Koopman modes analysis of sound generation in mixing layers

pressure with coherent structure events in a $2 \mathrm{D}$ compressible plane mixing layer. Int. $J$. Num. Meth. in Fluids, 62:906-926, 2010.

${ }^{15}$ W. S. Edwards, L. S. Tuckermann, R. A. Friesner, and D. C. Sorensen. Krylov methods for the incompressible navier-stokes equations. J. Comput. Phys., 110:82-102, 1994.

${ }^{16}$ U. Ehrenstein, P.-Y. Passaggia, and F. Gallaire. Control of a separated boundary layer: reduced-order modeling using global modes revisited. Theor. Comp. Fluid Dyn., 25(1):195207, 2011.

${ }^{17}$ X. Garnaud, L. Lesshafft, P.J. Schmid, and J.-M. Chomaz. A relaxation method for large eigenvalue problems, with an application to flow stability analysis. Journal of Computational Physics, 231(10):3912-3927, 2012.

${ }^{18}$ X. Garnaud, L. Lesshafft, P.J. Schmid, and P. Huerre. Modal transient dynamics of jet flows. Physics of Fluids., 25:044103, 2013.

${ }^{19}$ X. Gloerfelt, C. Bailly, and D. Juvé. Direct computation of the noise radiated by a subsonic cavity flow and application of integral methods. J. Sound Vib., 226(1):119-146, 2003.

${ }^{20} \mathrm{X}$. Gloerfelt and T. Le Garrec. Generation of inflow turbulence for aeroacoustic applications. In 14th AIAA/CEAS Aeroacoustics Conference 15-17 May, Vancouver, Canada, AIAA Paper 2008-2926, 2008.

${ }^{21} \mathrm{X}$. Gloerfelt and P. Lafon. Direct computation of the noise induced by a turbulent flow though a diaphragm in a duct at low Mach number. Comput. Fluids, 37:388-401, 2008.

${ }^{22}$ M. Grilli, P.J. Schmid, S. Hickel, and N.A. Adams. Analysis of unsteady behaviour in shockwave turbulent boundary layer interaction. J. Fluid Mech., 700:16-28, 2012.

${ }^{23}$ C.-M. Ho and N. S. Nosseir. Dynamics of an impinging jet. part 1. the feedback phenomenon. J. Fluid Mech., 105:119-142, 1981.

${ }^{24}$ T. L. Jackson and C. E. Grosch. Inviscid spatial stability of a compressible mixing layer. J. Fluid Mech., 208:609-637, 1989.

${ }^{25}$ A. Kierkegaard, E. Åkervik, G. Efraimsson, and D.S. Henningson. Flow field eigenmode decompositions in aeroacoustics. Computers and Fluids, 39:338-344, 2010.

${ }^{26}$ G.R. Kirchhoff. Towards a theory of light rays. Annalen der Physik und Chemie, 18:663695, 1883.

${ }^{27}$ M. Lesieur, P. Comte, E. Lamballais, O. Métais, and G. Silvestrini. Large-eddy simulations of shear flows. Journal of Engineering Mathematics, 32:195-215, 1997.

${ }^{28}$ L. Lesshafft. Nonlinear global modes and sound generation in hot jets. PhD thesis, Ecole 
Global and Koopman modes analysis of sound generation in mixing layers

polytechnique, 2006.

${ }^{29}$ L. Lesshhaft, P. Huerre, and M. Terracol. Nonlinear global modes in hot jets. J. Fluid Mech., 554:393-409, 2006.

${ }^{30}$ C. J. Mack and P.J. Schmid. Global stability of swept flow around a parabolic body: features of the global spectrum. J. Fluid Mech., 669:375-396, 2011.

${ }^{31}$ I. Mezic. Analysis of fluid flows via spectral properties of the Koopman operator. Annu. Rev. Fluid Mech., 45:357-378, 2012.

${ }^{32}$ P. J. Morris, L. N. Long, A. Bangalore, and Q. Wang. A parallel three-dimensional computational aeroacoustics method using nonlinear disturbance equations. J. Comput. Phys, 133:56-78, 1997.

${ }^{33}$ P. J. Morris, L. N. Long, and T. E. Scheidegger. Parallel computations of high speed jet noise. In 5th AIAA/CEAS Aeroacoustics Conference, 10-12 May, Greater Seattle, WA, AIAA Paper 99-1873, 1999.

${ }^{34}$ R. Moser and M. Rogers. The three-dimensional evolution of a plane mixing layer: pairing and transition to turbulence. J. Fluid Mech., 247:275-320, 1993.

${ }^{35}$ J.W. Nichols and S.K. Lele. Global modes and transient response of a cold supersonic jet. J. Fluid Mech., 669:225-241, 2011.

${ }^{36} \mathrm{~S}$. A. Ragab and J. L. Wu. Linear instabilites in two-dimensional compressible mixinglayer. Phys. Fluids A, 1(6):957-966, 1989.

${ }^{37}$ J. Ristorcelli and G. Blaisdell. Consistent initial conditions for the DNS of compressible turbulence. Physics of Fluids, 9:4-6, 1997.

${ }^{38}$ C.W. Rowley, I. Mezic, S. Bagheri, P. Schlatter, and D.S. Henningson. Spectral analysis of nonlinear flows. J. Fluid Mech., 641:115-127, 2009.

${ }^{39}$ N. D. Sandham and W. C. Reynolds. Three-dimensional simulations of large eddies in the compressible mixing layer. J. Fluid Mech., 224:133-158, 1991.

${ }^{40}$ T. Sayadi, J.W. Nichols, P.J. Schmid, and M.R. Jovanovic. Dynamic mode decomposition of h-type transition to turbulence. Center for Turbulence Research proceedings, 2012.

${ }^{41}$ P. J. Schmid. Dynamic mode decomposition of numerical and experimental data. J. Fluid Mech., 656:5-28, 2010.

${ }^{42}$ P. J. Schmid and D. S. Henningson. Stabiblity and transition in shear flows. Springer, 2001.

${ }^{43}$ P.J. Schmid, L. Li, M.P. Juniper, and O. Pust. Applications of the dynamic mode decom- 
Global and Koopman modes analysis of sound generation in mixing layers

position. Theor. Comp. Fluid Dyn., 1-4(25):249-259, 2011.

${ }^{44}$ J. M. Seiner, M. K. Ponton, and T. R. S. Bhat. Mach wave emission from a hightemperature supersonic jet. AIAA J., 32(12):2345-2350, 1994.

${ }^{45}$ D. Sipp and A. Lebedev. Global stability of base and mean flows: a general approach and its applications to cylinder and open cavity flows. J. Fluid Mech., 593:333-358, 2007.

${ }^{46}$ P. J. Strykowski and D. L. Niccum. The stability of countercurrent mixing layer in circular jet. J. Fluid Mech., 227:309-343, 1991.

${ }^{47}$ C. K. W. Tam and D. E. Burton. Sound generated by instability waves of supersonic flows. Part 1. Two-dimensional mixing layer. J. Fluid Mech, 138:249-271, 1984.

${ }^{48}$ C. K. W. Tam and Z. Dong. Radiation and outflow boundary conditions for direct computation of acoustic and flow disturbances in a nonuniform mean flow. J. Comput. Phys, 4(2):175-201, 1996.

${ }^{49}$ C. K. W. Tam and P. J. Morris. The radiation of sound by the instability waves of a compressible plane turbulent shear layer. J. Fluid Mech, 98(2):349-381, 1980.

${ }^{50}$ C. K. W. Tam and J. C. Webb. Dispersion-relation-preserving finite difference schemes for computational acoustics. J. Comput. Phys, 107(2):262-281, 1993.

${ }^{51} \mathrm{~V}$. Theofilis. Advances in global linear instability of nonparallel and three-dimensional flows. Progress in Aerospace Sciences, 39(4):249-315, 2003.

${ }^{52} \mathrm{~V}$. Theofilis. Global linear instability. Annual Reviews of Fluid Mechanics, 43:319-352, 2011.

${ }^{53}$ S. Yamouni, D. Sipp, and L. Jacquin. Interaction between feedback aeroacoustic and acoustic resonance mechanisms in a cavity flow: a global stability analysis. J. Fluid Mech., 717:134-165, 2013. 